\title{
TU/e EN⿴HONE

\section{Effect of linear mixing in EEG on synchronization and complex network measures studied using the Kuramoto model}

\section{Citation for published version (APA):}

Ahmadi, N., Pei, Y., \& Pechenizkiy, M. (2019). Effect of linear mixing in EEG on synchronization and complex network measures studied using the Kuramoto model. Physica A: Statistical Mechanics and its Applications, 520, 289-308. https://doi.org/10.1016/j.physa.2019.01.003

\section{Document license:}

TAVERNE

DOI:

10.1016/j.physa.2019.01.003

Document status and date:

Published: 15/04/2019

\section{Document Version:}

Publisher's PDF, also known as Version of Record (includes final page, issue and volume numbers)

\section{Please check the document version of this publication:}

- A submitted manuscript is the version of the article upon submission and before peer-review. There can be important differences between the submitted version and the official published version of record. People interested in the research are advised to contact the author for the final version of the publication, or visit the $\mathrm{DOI}$ to the publisher's website.

- The final author version and the galley proof are versions of the publication after peer review.

- The final published version features the final layout of the paper including the volume, issue and page numbers.

Link to publication

\section{General rights}

Copyright and moral rights for the publications made accessible in the public portal are retained by the authors and/or other copyright owners and it is a condition of accessing publications that users recognise and abide by the legal requirements associated with these rights.

- Users may download and print one copy of any publication from the public portal for the purpose of private study or research.

- You may not further distribute the material or use it for any profit-making activity or commercial gain

- You may freely distribute the URL identifying the publication in the public portal.

If the publication is distributed under the terms of Article 25fa of the Dutch Copyright Act, indicated by the "Taverne" license above, please follow below link for the End User Agreement:

www.tue.nl/taverne

Take down policy

If you believe that this document breaches copyright please contact us at:

openaccess@tue.nl

providing details and we will investigate your claim. 


\title{
Effect of linear mixing in EEG on synchronization and complex network measures studied using the Kuramoto model
}

\author{
Negar Ahmadi *, Yulong Pei, Mykola Pechenizkiy \\ Department of Mathematics and Computer Science, Eindhoven University of Technology, The Netherlands
}

\section{H I G H L I G H T S}

- Investigating the effect of volume conduction on synchronization between EEG signals

- Comparing the sensitivity of common synchronization measures to the presence of the volume conduction effect.

- Investigating the effect of volume conduction on various complex networks measures.

\section{A R T I C L E I N F O}

\section{Article history:}

Received 11 February 2018

Received in revised form 3 December 2018

Available online 11 January 2019

\section{Keywords:}

EEG

Volume conduction

Synchronization

Network measures

Kuramoto model

\begin{abstract}
A B S T R A C T
Volume conduction in the brain may influence the synchronization between EEG signals considerably, as it may lead to detection of spurious functional couplings among the recording channels. It has been shown that the volume conduction effect can be approximated as a linear mixing of the electrical fields of the brain regions. In this paper, we investigate the reliability of various synchronization measures in the presence of the linear superposition in EEG time series. For this purpose, we applied linear mixing to artificially generated EEG times series using the Kuramoto model of coupled phase oscillators, which represents the behavior of coupled systems with local interactions at the fundamental level. Our simulation results showed that the phase-lag index and the synchronization measures based on the visibility graph algorithms were less sensitive to the linear mixing effect and could predict the coupling degree correctly even with strongly overlapping signals. The results of our further data analyses demonstrated the effect of linear superposition in time series on the behavior of various complex network measures. For each case, we provide recommendations for proper choices of synchronization measures to obtain complex network characteristics that are minimally sensitive to linear mixing.
\end{abstract}

(c) 2019 Elsevier B.V. All rights reserved.

\section{Introduction}

The human brain is the most complex system yet discovered, and understanding its function is one of the greatest scientific challenges. A functional brain network, which is constructed through interconnections among brain regions according to some measure of functional connectivity, is the statistical dependencies among the activity of brain regions [1,2]. In order to build a brain network and estimate its functional connectivity, electroencephalography (EEG) technology is commonly used, due to its high temporal resolution (milliseconds), which helps to detect brief neuronal events [3]. For this purpose, the locations of the EEG sensors serve as nodes and the link (or connection) between any two nodes represents the

\footnotetext{
* Correspondence to: P.O.Box: 513, 5600MB, TU/e, Eindhoven, The Netherlands.

E-mail address: n.ahmadi@tue.nl (N. Ahmadi).
} 


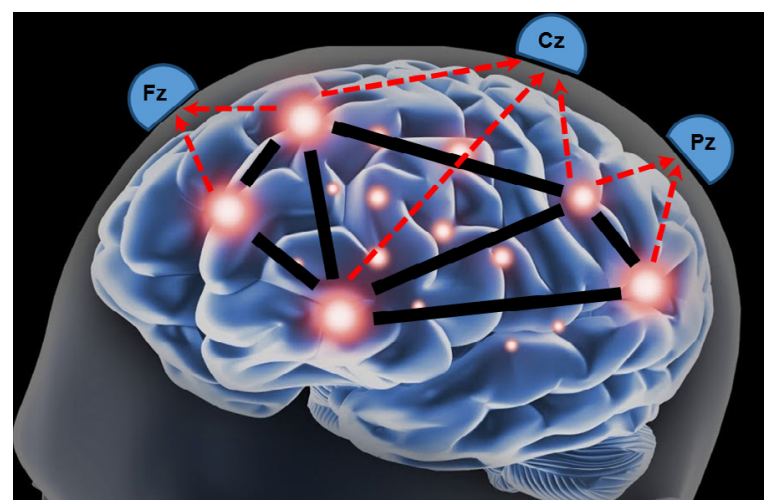

Fig. 1. Schematic effect of volume condition complication on EEG signal recording. The dashed arrows show the activity of each region (filled circles) that is recorded by the EEG recording terminal and the thick lines represent the correlation among the regions.

magnitude of the correlation between the EEG time series associated with these nodes [1,4-6]. The properties of the brain's functional networks are linked to their functions and can be affected by neurological diseases; for example, Alzheimer's disease is often associated with a loss of local and global correlated activity among brain regions [7].

However, recorded EEG time series suffer from the volume conduction (VC) effect [8,9]. In EEG recording, electrodes are used to record brain neuronal activity, i.e., electrical events generated several centimeters below the recording electrodes. Hence, the recording electrode is not in direct contact with the desired brain region, and there is some medium separating the two, including brain matter, cerebrospinal fluid, dura, skull, and scalp. Due to the conductivity of the separating medium, electrical currents spread through different resistive layers, providing a distorted view of brain activity at the scalp [8]; this phenomenon induces a blurring effect when recording at scalp level. Consequently, each EEG channel records the instantaneous linear mixing of multiple brain source activities instead of the activity of just the brain source in its vicinity $[10,11]$ (see Fig. 1). As a result, the VC effect may lead to the detection of spurious functional correlations among EEG time series, even if all the brain sources are independent $[12,13]$.

In this paper, we study the effects of VC on synchronization and network measures. To achieve this goal, after the generation of artificial EEG time series using the original Kuramoto model, the VC effect was implemented using a linear superposition formula. By applying selected synchronization measures on the generated time series, the corresponding functional networks (or weighted graphs) were constructed. Then, some complex network measures, including the clustering coefficient, betweenness, eigenvalue centrality, and the largest eigenvalue, were studied to understand the effect of the overlap on different patterns of the network.

The rest of this paper is organized as follows: The details of the method are presented in Section 2. In Section 3, we present our simulation study demonstrating the sensitivity of the synchronization measures to the linear mixing effect and the behavior of the complex network measures as a function of coupling strength. Discussion of the findings is presented in Section 4, followed by concluding remarks in Section 5.

\section{Methods}

In this section, we first present the model for EEG generation. Then, we present the construction of the functional network from some selected synchronization measures. Also, details of these selected measures are briefly presented. And finally, we present the details of the selected complex network measures.

\subsection{Model for EEG Generation}

We first present the mathematical formulation of the VC problem. Then, we introduce the classical Kuramoto model, which was used as a method of generating EEG time series.

\subsubsection{Volume conduction problem}

In order to understand the relationship between EEG time series and the underlying primary source configuration, various techniques (such as the concentric-spheres model [14]) can be used. The dipole approximation to cortical current sources provides a basis for any realistic source model of EEG. It is based on the idea that at large distances, any complex current distribution in a small region of the cortex can be approximated by a dipole moment per unit volume, $\vec{P}(\vec{r}, t)\left[\right.$ A.s $\left./ \mathrm{mm}^{2}\right]$, which is a vector field and is also called the polarization density [15]. The strength of the dipole moment depends not only on the strength of the individual sources, but also on their spatial distribution within the tissue mass. The relationship between the scalar potential of the scalp, $\Phi(\vec{r}, t)$ and $\vec{P}(\vec{r}, t)$, can be written in terms of the vector Green,s function $\vec{G}_{E}\left(\vec{r}, \vec{r}^{\prime}\right)$ that describes 
the head volume conductor. In other words, the scalp potential may be approximated by the following integral over the cortical volume $V$ :

$$
\Phi(\vec{r}, t)=\iiint_{V} \vec{G}_{E}\left(\vec{r}, \overrightarrow{r^{\prime}}\right) \cdot \vec{P}\left(\vec{r}^{\prime}, t\right) d V\left(\vec{r}^{\prime}\right)
$$

where $d V\left(\overrightarrow{r^{\prime}}\right)$ represents the volume element. Here, the Green,s function for concentric spheres [14] depends only on the properties of VC in the head and expresses the relationship between a unit source at location $\vec{r}^{\prime}[\mathrm{mm}]$ and the measurement point on the scalp surface $\vec{r}[\mathrm{~mm}]$. The potential anywhere on the scalp surface is then expressed as an integral (or weighted sum) of contributions from all sources in the brain. The weight given to each source within the volume of the brain depends on the locations and conductivities of the different tissues in the head. However, if we assume all of the sources are in the cortex, the sources can be reasonably assumed to be oriented normal to the cortical surface. In this case, the above equation reduces to an integral over the surface of the brain, and the potential recorded by every EEG terminal on the scalp surface can be approximated as a linear superposition of electric fields from all sources in brain. The linear superposition of electric fields allows the assumption that each electrode records an EEG signal that is a linear mixture of all underlying sources of electrical activity.

\subsubsection{The Kuramoto model}

Denoted by $\theta_{i}(t)$, the phase of oscillator $i$ at discrete time $t$, the Kuramoto model obeys the following dynamical equation for a large network of $M$ globally coupled oscillators [16]:

$$
\frac{d \theta_{i}(t)}{d t}=\omega_{i}+\frac{1}{M} \sum_{m=1}^{M} K \sin \left(\theta_{m}(t)-\theta_{i}(t)\right),
$$

where $\omega_{i}[\mathrm{~Hz}]$ is the natural frequency of oscillator $i$, and $K[\mathrm{~Hz}]$ is the strength of the couplings between the oscillators. In actuality, the ith oscillator adjusts its phase velocity according to input from other oscillators through the coupling strength $K$.

Kuramoto [16] further introduced the order parameter $r(t)$ as follows, which allows this model to be solved exactly when $M \rightarrow \infty:$

$$
r(t) e^{i \psi(t)}=\left|\frac{1}{M} \sum_{m=1}^{M} e^{i \theta_{m}(t)}\right|,
$$

where $\psi(t)$ indicates the average phase. The order parameter $r(t)$ captures the degree of phase coherence in the system. If the phases are uniformly distributed in the range of $[0, \pi]$ (i.e., have large circular variance), then $r(t) \approx 0$, meaning that there is no synchrony among the oscillators. On the other hand, if all the oscillators rotate grouped into a synchronous cluster with the same average phase $\psi(t)$, then $r(t) \rightarrow 1$, meaning that the phases of all oscillators are perfectly locked, which describes zero phase lag synchronization.

Eq. (2) can be rewritten as follows by multiplying both sides of Eq. (3) by $e^{i \theta_{i}}$ and equating the imaginary parts:

$$
\frac{d \theta_{i}(t)}{d t}=\omega_{i}+K r \sin \left(\psi-\theta_{i}\right)
$$

where $r$ and $\psi$ are mean-field quantities. This formulation shows that the oscillators, equations are no longer explicitly coupled, which means that the phases $\theta_{i}$ evolve independently from each other. However, the interaction is actually set through $r$ and $\psi$. Note that the effective coupling is now proportional to the order parameter $r$, creating a feedback relation between coupling and synchronization. More specifically, small increments in the order parameter $r$ end up increasing the effective coupling in Eq. (4), thus attracting more oscillators to the synchronous group. From this process, a self-consistent relation between the phases $\theta_{i}$ and the mean-field is found, i.e., $r$ and $\psi$ will define the evolution of $\theta_{i}$, but at the same time, the phases $\theta_{i}$ self-consistently yield the mean-field through Eq. (3).

In the limit $M \rightarrow \infty$, the relation between the order parameter $r$ and coupling strength $K$ is given by [16]

$$
\left\{\begin{array}{l}
\text { if } K<K_{c}: \quad r=0, \\
\text { if } K \geq K_{c}: \quad r=\sqrt{1-\left(K_{c} / K\right)} .
\end{array}\right.
$$

Kuramoto showed that if the oscillators, natural frequencies $\omega_{i}$ are distributed around a central frequency $\omega_{0}$ spread by some value $\gamma[\mathrm{Hz}]$ according to a Lorentzian density:

$$
g\left(\omega_{i}\right)=\frac{\gamma}{\pi\left[\gamma^{2}+\left(\omega_{i}-\omega_{0}\right)^{2}\right]}
$$

then the critical value reads $K_{c}=2 \gamma$. This value indicates that the oscillators are desynchronized completely, and $r=0$ until the coupling strength $K$ exceeds a critical value $K_{c}$. When $K \geq K_{c}$, the population splits into a partially synchronized state consisting of two groups of oscillators: a desynchronized group and a synchronized group that contributes to the order parameter $r$. With further increase in $K$, more and more oscillators are recruited into the synchronized group, and $r$ grows accordingly. 


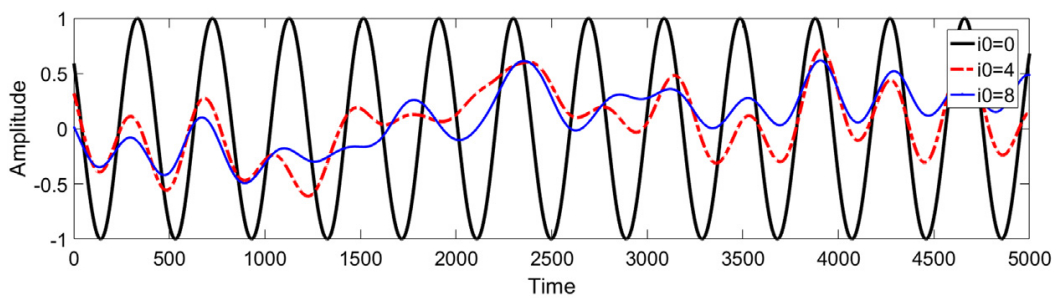

(a)

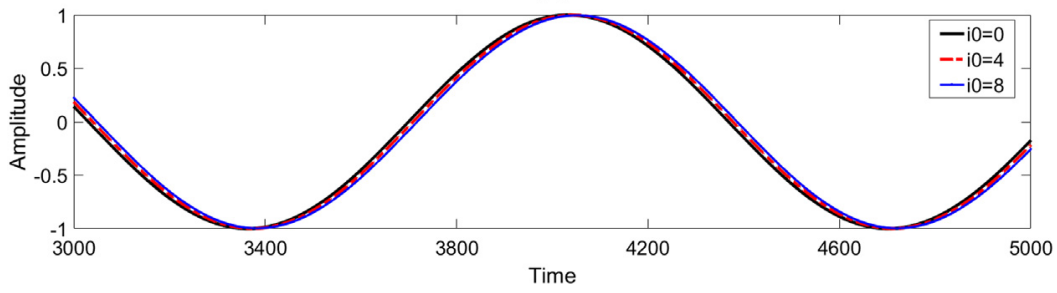

(b)

Fig. 2. Generated EEG signal from an identical oscillator with contribution of different sources when (a) $K=0$ and (b) $K=8$.

\subsubsection{Generating EEG signals}

Theoretically, an infinite number of oscillators (i.e., $M \rightarrow \infty$ ) are necessary for the analytical results to hold. However, it has been shown that with only 64 oscillators, the model can explain various empirical results quite well [17]. Therefore, we solved the Kuramoto model for $M=64$ in our work. The time series corresponding to oscillator $i$ at time $t$ was obtained as

$$
O_{i}(t)=A \sin \theta_{i}(t)
$$

where, for simplicity, a constant amplitude $A=1$ was set for all time series. As mentioned earlier, the VC produces an instant zero-lag correlation among the brain sources, and it is commonly represented as a linear mixture of the sources [18]. In order to add the VC effect to the model, we generated 64 EEG time series with mean frequency $10 \mathrm{~Hz}$ (corresponding to the alpha band) and different degrees of overlap. Also, for simplicity, we set $\gamma=1$. Then, the voltage $y_{i}(t)$ of the $i$ th EEG channel at time $t$ was defined as [19]

$$
y_{i}(t)=\frac{1}{2 i_{0}+1} \sum_{m=i-i_{0}}^{m=i+i_{0}} O_{m}(t),
$$

where the so-called overlapping parameter $i_{0}=0,4,8$ determines the contribution of multiple sources to each EEG channel, and $2 i_{0}$ is the number of shared oscillators for consecutive EEG channels. Note that a periodic boundary condition is imposed in Eq. (8), i.e., $\theta_{i+M}=\theta_{i}$ and $O_{i+M}=O_{i}$.

Fig. 2 shows the EEG time series of an identical oscillator with $i_{0}=0$ and 8 for weak and strong coupling strengths, respectively. One can see that for vanishing coupling strength (i.e., $K=0$ ), the generated signals show random behavior due to the phase delay among oscillators. However, by increasing the coupling strength, the signals become similar and synchronize quite well.

It is clear that Eq. (8) is a simple mixing formulation, as several time series are added together in a linear fashion to represent the VC effect. According to the literature, there are various and more complex techniques to capture the VC effect more realistically $[20,21]$. However, applying a linear mixing equation such as Eq. (8) is fairly reasonable to demonstrate the VC effect at a fundamental level [19,22], which is the purpose of this work.

It is worth mentioning that in order to capture true brain activity and discover the complex patterns of relationship among brain regions, it is necessary to record brain waves with a high resolution; otherwise, significant parts of the brain activity may be lost. EEG techniques using high temporal resolution allow the capture of neural events on the order of milliseconds. Fig. 3 shows a sample of EEG data recorded with two different time samplings, 1/128 and 1/32 s [23]. It is evident that EEG recordings differ as a result of changes in resolution. Accordingly, the resultant brain network recorded by EEGs with low resolution will be different from that of EEGs with high resolution. Therefore, in this work, the Kuramoto model (i.e., Eq. (2)) was numerically solved using the Euler integration method in timesteps of $2 \mathrm{~ms}$, corresponding to a sample frequency $500 \mathrm{~Hz}$. The simulations were performed with the generating model for 64 oscillators and $K$ ranging from 0 to 8 in steps of 0.5. In all simulations, time series with $N=10000$ samples were generated using Eq. (8). However, the initial 5000 samples were discarded to eliminate transients. Then, the resulting time series were subjected to synchronization analysis by applying different techniques (see Section 2.2). For each value of $i_{0}$ and $K$, five trials were completed and the final value of synchronization was calculated by averaging over all five trials. In this study, the software MATLAB Version R2017 was used for programming purposes. 


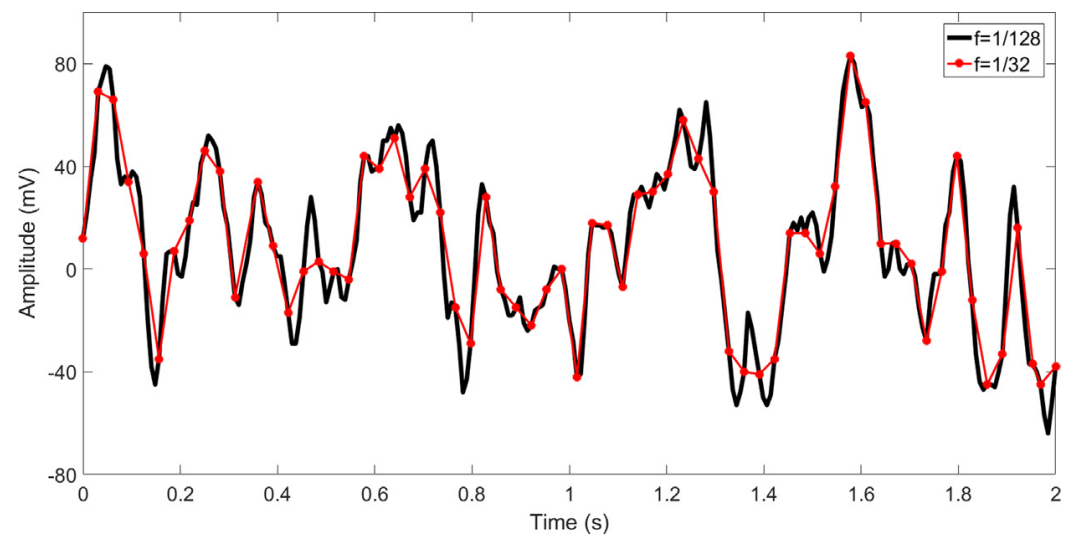

Fig. 3. EEG recordings with two different temporal resolutions.

\subsection{Construction of a functional network from synchronization measures}

In order to create a functional network (or weighted graph), a matrix containing the oscillator pairwise correlations is required. Thus, one needs to calculate the correlations among all pairs of time series and deduce the respective correlation (or adjacency) matrix. A functional network is a mathematical representation of the brain and is defined by a collection of nodes and links between pairs of nodes. Nodes in a functional brain network represent brain regions, while links represent functional connections corresponding to the magnitude of the temporal correlation between node pairs. In this section, we present some selected synchronization measures that were used to estimate correlations between all pairs of time series. Each of the presented synchronization measures results in the calculation of a correlation matrix, which can also be regarded as a weighted undirected graph.

\subsubsection{Cross-correlation (CC)}

The cross-correlation (CC) function measures the linear correlation between two time series as a function of their delay time, which is of interest because such a time delay may reflect a causal relationship between the time series. The CC between two time series $x(t)$ and $y(t)$ with the same $N$ samples length, where $t$ denotes discrete time $(t=1, \ldots, N)$, is expressed as [24]

$$
\mathrm{CC}=C_{x y}(h)=\frac{1}{N-h} \sum_{t=1}^{N-h} x(t+h) y(t),
$$

where $t=1, \ldots, N$ denotes discrete time and $h \in\{-(N-1), \ldots, 0, \ldots, N-1\}$ denotes time lag. Here, CC $= \pm 1$ presents the complete linear direct and inverse correlations, respectively, and $\mathrm{CC}=0$ indicates lack of linear correlation for a given time lag.

\subsubsection{Coherence (Coh) \& imaginary part of coherence (ImPC)}

To compute the coherence, it is necessary to know the instantaneous phase and amplitude of the two time series involved. For this purpose, either linear or nonlinear techniques can be used as two conceptually distinct methods to extract the phase and amplitude of time series. Linear techniques, such as the Fourier transform, assume a constant phase and amplitude within the estimation window. Nonlinear techniques measure time-dependent instantaneous phase and amplitude, which illustrate the moment-to-moment change in time series. The Hilbert transform is a well-known linear technique for obtaining the instantaneous phase and amplitude of time series. The Hilbert transform of a real valued time series $x(t)$ is obtained as

$$
x_{h}(t)=\frac{1}{\pi} P V \int_{-\infty}^{\infty} \frac{x(\tau)}{t-\tau} d \tau,
$$

where $P V$ refers to the Cauchy principle value. The Hilbert transform is simply a $\pi / 2$ shift in the phase of the original signal and does not alter the spectral distribution. In other words, it can be computed by performing a Fourier transform, shifting all the phases by $\pi / 2$, then performing an inverse Fourier transform. Hence, we follow the analytical signal approach as presented below to determine the instantaneous phase and amplitude of two time series $x(t)$ and $y(t)$ as [25]

$$
\begin{aligned}
& z_{x}(t)=x(t)+i x_{h}(t)=A_{x}(t) e^{i \phi_{x}(t)} \\
& z_{y}(t)=y(t)+i y_{h}(t)=A_{y}(t) e^{i \phi_{y}(t)},
\end{aligned}
$$


where $i$ is the imaginary unit and $y_{h}(t)$ is the Hilbert transform of time series $y(t)$. The instantaneous phase $\phi(t)$ and amplitude $A(t)$ of the time series is obtained as the argument and magnitude of the analytic extension as follows:

$$
\begin{aligned}
& \phi_{x}(t)=\arctan \frac{x_{h}(t)}{x(t)}, A_{x}(t)=\sqrt{[x(t)]^{2}+\left[x_{h}(t)\right]^{2}}, \\
& \phi_{y}(t)=\arctan \frac{y_{h}(t)}{y(t)}, A_{y}(t)=\sqrt{[y(t)]^{2}+\left[y_{h}(t)\right]^{2}} .
\end{aligned}
$$

The complex-valued coherence between two time series $x(t)$ and $y(t)$ is defined as the cross spectrum divided by the product of the two power spectra [26]. Its mean over all frequencies can alternatively be computed via the mean over time of the corresponding analytical time series as

$$
C=\frac{z_{x} z_{y}^{*}}{\left|z_{x}\right|\left|z_{y}\right|}=\frac{\left\langle A_{x}(t) A_{y}(t) e^{i \Delta \phi(t)}\right\rangle}{\left|A_{x}\right|\left|A_{y}\right|}=\mathrm{Coh}+i \mathrm{ImPC}
$$

where $z_{y}^{*}=A_{y}(t) e^{-i \phi_{y}(t)}$ is the complex conjugate of $z_{y},|\cdot|$ denotes the absolute value, and $\langle\cdot\rangle$ denotes averaging over time with time interval $\Delta t=2$ milliseconds. Also,

$$
\Delta \phi(t)=\left|\phi_{x}(t)-\phi_{y}(t)\right|
$$

is the instantaneous phase difference between the two time series at a specific frequency. The complex-valued coherence (see Eq. (15)) can be split into real and imaginary parts. The real value, which is typically referred to as coherence (Coh), is bounded between 0 and 1 . For a given frequency, $\mathrm{Coh}=0$ indicates that the activities of the time series in this frequency are linearly independent, whereas a value of $\mathrm{Coh}=1$ represents the maximum linear correlation for this frequency. When the complex-valued coherence is projected onto the imaginary axis, we can obtain the imaginary part of coherence (ImPC). The ImPC is only sensitive to synchronization of two oscillators which are time-lagged to each other. If volume conduction does not cause a time-lag, the ImPC is insensitive to artifactual self-interaction. In fact, the ImPC is insensitive to zero-lag effects, as it removes the contribution of the zero phase differences that, due to the complex exponentiation, gives real phase-locking values [26].

\subsubsection{Phase Coherence (PC)}

The first step in phase synchronization is the extraction of instantaneous phases from the time series using the Hilbert transform (see Eq. (13)). Next, the instantaneous phase difference is calculated by Eq. (16). In order to determine whether this phase difference is bounded, the notation of phase coherence (PC) described by Mormann et al. [27] is used. This notation resembles the conventional statistics for circular or directional data. Hence, instantaneous phase differences are projected on the unit circle, and the length $R$ of the average resultant vector is calculated as

$$
\mathrm{PC}=R=\left\langle e^{i \Delta \phi(t)}\right\rangle=\left|\frac{1}{N} \sum_{t=0}^{N-1} e^{i \Delta \phi(t)}\right|,
$$

where $\langle\cdot\rangle$ denotes averaging over all time samples, $t$ denotes discrete time $(t=1, \ldots, N)$, and $N$ is the number of time samples. The value of $R$ ranges from 0 to 1 , where in the case of perfect phase locking we have $R=1$, whereas in the case of a random distribution of phases on the unit circle, $R$ will tend to 0 . By construction, $R$ only depends upon the phase relations between the time series and is not sensitive to the amplitude of the time series.

\subsubsection{Phase lag index (PLI)}

The phase lag index (PLI) is calculated as [19]

$$
\mathrm{PLI}=|\langle\operatorname{sign}[\Delta \phi(t)]\rangle|
$$

If no phase coupling exists between two time series, then the phase difference distribution $\Delta \phi(t)$ is expected to be flat. Therefore, any deviation from this flat distribution indicates phase synchronization. The PLI ranges between 0 (i.e., no coupling) and 1 (which means a perfect phase locking).

\subsubsection{Synchronization likelihood (SL)}

The synchronization likelihood (SL) measure computes the number of similar patterns in the time series that are repeated simultaneously [28]. For each single time series $x(t)$, a state vector is reconstructed as

$$
X(t)=(x(t), x(t+l), x(t+2 l), \ldots, x(t+(D-1) l)),
$$

where $(t=1, \ldots, N)$ denotes discrete time, $l$ is the time lag, and $D$ is the embedding dimension. Here, the time lag is chosen such that the highest frequency is sampled at least twice per cycle, and the embedding dimension such that at least one whole cycle of the slowest oscillation is captured. More specifically, the $l$ and $D$ parameters are set to $l=f_{s} / 3 f_{h}$ and $D=\left(3 f_{h} / f_{l}\right)+1$, respectively, where $f_{s}$ is the sampling frequency, $f_{l}$ is the lowest frequency, and $f_{h}$ is the highest frequency 
of the content data $[29,30]$. Here, $X(t)$ represents the state of the system in a time interval of length $(D-1) l$, with $t$ as the beginning time instant.

For each time instant $t, P_{X(t)}^{\varepsilon_{X}(t)}$ is defined as the probability that the state vectors are closer to each other than critical distance $\varepsilon_{X}(t)$

$$
P_{X(t)}^{\varepsilon_{X}(t)}=\frac{1}{2\left(w_{2}-w_{1}\right)}\left[\sum_{j=-w_{2}}^{j=-w_{1}} D_{X}(t)+\sum_{j=w_{1}}^{j=w_{2}} D_{X}(t)\right],
$$

where

$$
D_{X}(t)=\theta\left(\varepsilon_{X}(t)-|X(t)-X(t+j)|\right) .
$$

Here $\theta$ is a Heaviside step function, $\theta(x)=0$ if $x \leq 0, \theta(x)=1$ for $x>0$, and the sign $|\cdot|$ indicates the Euclidean distance between the state vectors, which is calculated by the Pythagorean formula. The $w_{1}=2(D-1) l$ parameter establishes an exclusion window around the time instant $i$ and is used to set a lower band for time instant $t$, to give the system time to evolve into a different state. On the other hand, the inclusive window parameter $w_{2}$ is used to control the time resolution of the synchronization measure by setting an upper band to the $t$ values. These windows are chosen such that $w_{1} \leq w_{2} \leq N$. Theoretically, $w_{2}$ could be chosen as the length of the entire time series, which might not be feasible for large datasets, due to the expensive computational cost. The combination of $w_{1}$ and $w_{2}$ establishes the surrounding of $t$ given by the subintervals $\left[t-w_{2}, t-w_{1}\right)$ and $\left(t+w_{1}, t+w_{2}\right]$.

The critical distance $\varepsilon_{X}(t)$ is determined by setting $P_{X(t)}^{\varepsilon_{X}(t)}=p_{\text {ref }}$, where $p_{r e f} \ll 1$. The reference probability parameter, $p_{\text {ref }}$, represents the fraction of state vectors inside the time subintervals $\left[t-w_{2}, t-w_{1}\right)$ and $\left(t+w_{1}, t+w_{2}\right]$, which are to be considered closer than the critical distance to $X(t)$. The value of $p_{\text {ref }}$ is set at an arbitrarily low level and does not depend on the properties of the time series, nor is it influenced by the embedding parameters [28]. In this work, we set $p_{\text {ref }}=0.01$, which means that one percent of the vectors $X(t+j)$ were considered recurrences of $X(t)$. The relationship between $w_{2}, p_{\text {ref }}$, and the number of recurrences, $n_{\text {rec }}$, was obtained by $n_{\text {rec }}=\left(w_{2}-w_{1}+1\right) \times p_{\text {ref }}$ [29].

Next, the synchronization likelihood between the state vectors of two time series, i.e., $X(t)$ and $Y(t)$, is determined as

$$
S_{X Y}(t)=\frac{1}{\left(w_{2}-w_{1}+1\right) \times p_{\text {ref }}}\left[\sum_{j=-w_{2}}^{j=-w_{1}} D_{X Y}(t)+\sum_{j=w_{1}}^{j=w_{2}} D_{X Y}(t)\right],
$$

where

$$
D_{X Y}(t)=\theta\left(\varepsilon_{X}(t)-|X(t)-X(t+j)|\right) \cdot \theta\left(\varepsilon_{Y}(t)-|Y(t)-Y(t+j)|\right) .
$$

The overall SL value is the average of $S_{X Y}(t)$ values over the all-time instants. Values of the SL are between a small number close to 0 , when there is no coupling, and 1 , in the case of a fully synchronized time series.

\subsubsection{Visibility graph-based methods}

The idea of mapping time series as graphs seems attractive because it lays a bridge between nonlinear signal analysis and complex networks theory. Hence, the visibility graph (VG) algorithm has attracted attention as a technique capable of being used for time series analysis due to its intrinsic non-locality, low computational cost, simple rules, and straightforward implementation. Visibility algorithms are a family of methods that map time series as graphs nonlinearly. Therefore, the tools of graph theory can be used for the characterization of time series. The VG-based algorithms are invariant under rescaling of both horizontal and vertical axes, and it has been shown that many time series' structural properties (e.g., periodicity, fractality, etc.) are inherited by the resultant visibility graph [31-33]. For purposes of synchronization, the series of the connectivity degree (i.e., the number of edges connected to a node) of the visibility graph nodes is considered a new time series, which is called the degree sequence (DS) time series. The measure of the synchronization between two DSs is called the VG similarity and can be presented as an alternative measure of synchronization between time series [31,32].

Let $x(t)$ be a univariate time series of $N$ discrete data $(t=1,2, \ldots, N)$. The VG algorithm converts the time series $x(t)$ to a graph, as a data point $x(t)$ is mapped into a node in the graph. The time point (i.e., a point on the time series) represents a moment in which the data is recorded (see Fig. 4(a)). By applying the original/horizontal visibility graph algorithm (OVG/HVG), an EEG time series of size $N$ maps to a visibility graph with $N$ nodes (see Figs. 4 (b) and 4(c)).

The original visibility graph (OVG) algorithm [31] implies that two arbitrary data points $\left[t^{*}, x\left(t^{*}\right)\right]$ and $\left[t^{\star}, x\left(t^{\star}\right)\right]$ have visibility, and consequently are two connected nodes of the associated graph, if any other data point $[t, x(t)]$ placed between them $\left(t^{*}<t<t^{\star}\right)$ satisfies

$$
x(t)<x\left(t^{*}\right)+\left[x\left(t^{\star}\right)-x\left(t^{*}\right)\right] \frac{t-t^{*}}{t^{\star}-t^{*}} \text { for all } t \text { such that }\left(t^{*}<t<t^{\star}\right) .
$$

The schematic of the above geometric criterion and its associated visibility graph are shown in Fig. 4(b). It is clear that two arbitrary data nodes at $t^{*}$ and $t^{\star}$ in the graph are connected if one can draw a straight line in the time series joining $x\left(t^{*}\right)$ and $x\left(t^{\star}\right)$ that does not intersect any intermediate data height. 
The so-called horizontal visibility graph (HVG), which is defined as a subgraph of the OVG, is obtained by restricting the visibility criterion and imposing horizontal visibility instead [32]. In the HVG algorithm, two arbitrary data nodes $t^{*}$ and $t^{\star}$ in the graph are connected if:

$$
x\left(t^{*}\right)>x(t) \text { and } x\left(t^{\star}\right)>x(t) \text { for all } t \text { such that: }\left(t^{*}<t<t^{\star}\right) .
$$

According to the HVG geometric criterion, two data points are connected if one can draw a horizontal line in the time series joining them that does not intersect any intermediate data height (see Fig. 4(c)).

Therefore, by applying the OVG/HVG, a time series of size $N$ maps to a graph with $N$ nodes, as the first node in Figs. 4(b) and 4(c) is associated with the first time point in Fig. 4(a). The second node corresponds to the second time point of the EEG time series, and so on.

After constructing the visibility graphs, the degree of each node is determined. The degree of node $t$ is the number of links connected to node $t$. Therefore, by counting the number of links that have node $t$ as an endpoint, we can determine the degree of each node. Then, by considering the degrees of all nodes, a DS time series is obtained. The corresponding DSs of the OVG and the HVG algorithms are shown in Fig. 4(d) as time series. Next, the synchronization of two time series $x(t)$ and $y(t)$ is determined through computation of correlation of the DSs of the corresponding visibility graphs as

$$
S_{x y}=\left|\frac{\operatorname{cov}\left[D S_{x}, D S_{y}\right]}{\sigma_{D S_{x}} \times \sigma_{D S_{y}}}\right|,
$$

where $D S_{(\cdot)}$ represents the degree sequence of a time series, $\operatorname{cov}[\cdot]$ is the covariance of time series, and $\sigma_{(\cdot)}$ is the standard deviation. The correlation values range from 0 to 1 , where $S_{x y}=0$ means the time series are not synchronized, and $S_{x y}=1$ means that the time series are identical [34,35].

\subsection{Network measures for characterizing local and global connectivity}

Functional brain networks are composed of many nodes and links, and as they rise in complexity, their comparison becomes challenging. However, various network measures can be used to analyze the networks and characterize one or more aspects of local or global brain connectivity. In this section, we describe some selected measures that can be used to detect aspects of the network.

\subsubsection{Clustering coefficient}

The clustering coefficient assesses the degree to which nodes tend to cluster together. In brain network studies, the clustering coefficient is considered to be a measure of the local connectivity of the functional brain network. Brain networks are small "worlds" in which different functional units can work independently but are connected to each other through hubs. A high clustering coefficient indicates the presence of local cliques forming specialized functional units. Given a weighted network $G$, the local clustering coefficient $c_{i}$ for node $i$ is defined as [36]

$$
c_{i}=\frac{2}{d_{i}\left(d_{i}-1\right)} \sum_{i, k}\left(\tilde{w}_{i j} \cdot \tilde{w}_{j k} \cdot \tilde{w}_{k i}\right)^{1 / 3},
$$

where $\tilde{w}_{i j}=w_{i j} / \max \left(w_{i j}\right)$ is the scaled weight. Here, $d_{i}\left(d_{i}-1\right) / 2$ is the maximum possible number of links when the subgraph of neighbors of node $i$ is completely connected. The global clustering coefficient for the whole graph is the average of the local values and is defined as [37]:

$$
C=\frac{1}{N} \sum_{i}^{N} c_{i},
$$

where $N$ is the number of nodes in the graph. It is clear that $0 \leq c_{i} \leq 1$ and $0 \leq C \leq 1$. Note that $c_{i}=1$ if node $i$ is the center of a fully interconnected cluster and $c_{i}=0$ if the neighbors of node $i$ are not connected to each other.

\subsubsection{Betweenness centrality}

Centrality refers to the relative importance of a vertex within the network. Mostly, the vertices in a network with higher centrality index values are perceived as being the more important vertices. Betweenness centrality quantifies the number of times that a node acts as a bridge along the shortest path between two other nodes. In an undirected network, a path between two nodes that has the minimum number of links is referred to as the shortest path between these two nodes. In the context of brain network analysis, a brain region (or EEG recording site) has a high betweenness centrality index if it is strategically located as a midpoint between several pairs of brain regions and, therefore, controls the flow of information across the brain network.

Consider an undirected graph $G=(V, E)$, where $V$ and $E$ denote its node and link set, respectively. For three distinct nodes $v_{1}, v_{2}, v_{3} \in V$, let $\sigma_{v_{1}, v_{3}} \neq 0$ be the number of shortest paths between $v_{1}$ and $v_{3}$ in $G$, and let $\sigma_{v_{1}, v_{3}}\left(v_{2}\right)$ be the number of shortest paths between $v_{1}$ and $v_{3}$ that pass through $v_{2}$. The betweenness centrality index of node $v_{2}$ is defined as [38]

$$
B\left(v_{2}\right)=\sum_{v_{1} \neq v_{2} \neq v_{3} \in V} \frac{\sigma_{v_{1}, v_{3}}\left(v_{2}\right)}{\sigma_{v_{1}, v_{3}}} .
$$




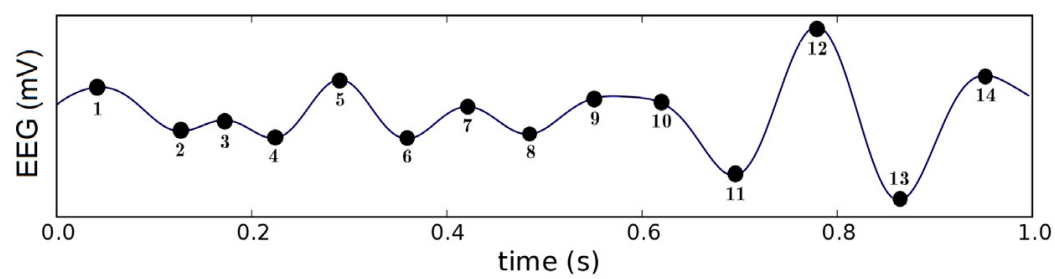

(a)
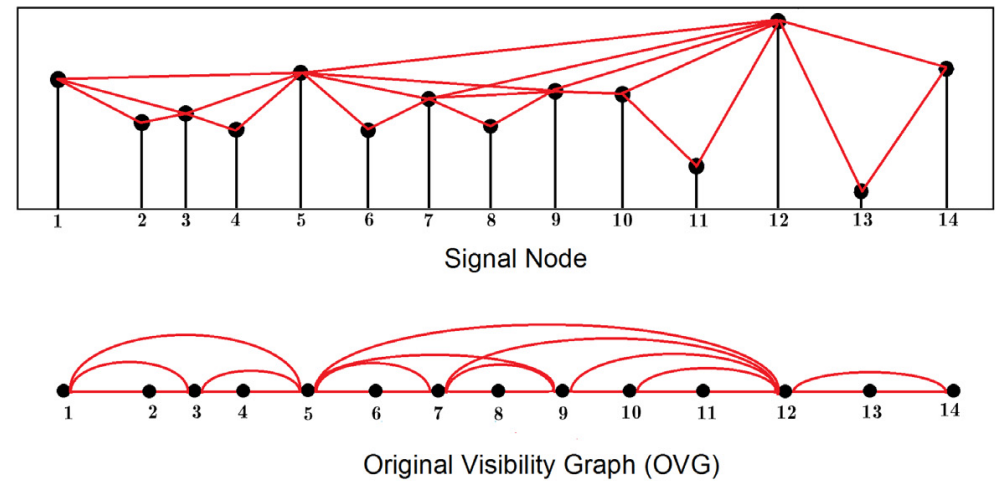

(b)

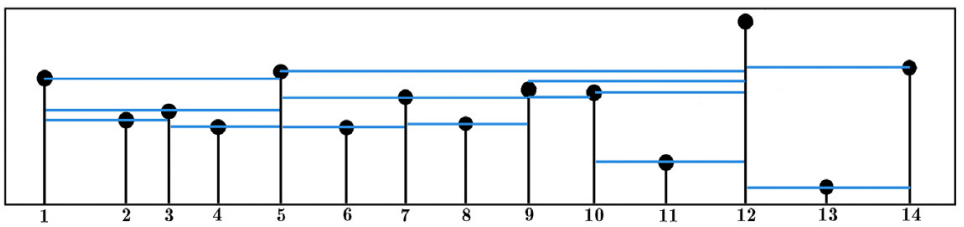

Signal Node

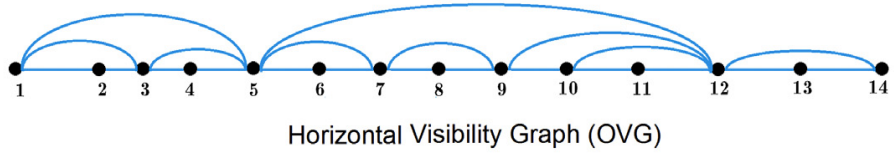

(c)

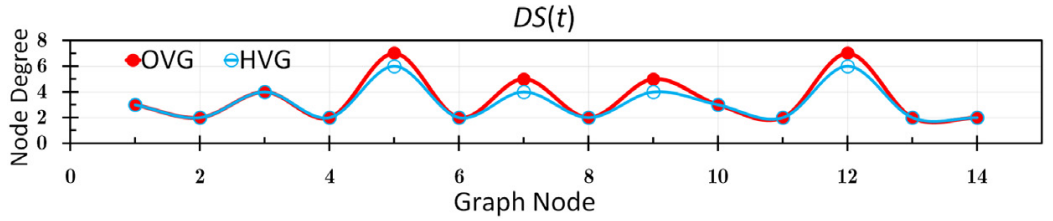

(d)

Fig. 4. (a) An EEG time series (filled circles represent time points), (b) top: applying OVG criteria on time points, bottom: corresponding visibility graph, (c) top: applying HVG criteria on time points, bottom: corresponding graph, and (d) corresponding degree sequences of the OVG and HVG for such time points.

The average node betweenness centrality of the graph is defined as follows:

$$
\bar{B}(G)=\frac{1}{N} \sum_{v_{2} \in V} B\left(v_{2}\right) .
$$

The betweenness centrality lies between zero and $\left(\begin{array}{c}N-1 \\ 2\end{array}\right)$, where the value 0 is obtained if and only if all neighbors of $v_{i}$ induce a maximal clique in $G$. 


\subsubsection{Eigenvector centrality and largest eigenvalue}

Eigenvector centrality is a global measure of centrality, as it does not focus on the immediate vicinity of nodes but instead considers all possible indirect connections. It operates under the premise that connections to nodes that are themselves well-connected should be given more weight than connections to less well-connected nodes. Eigenvector centrality for all nodes in the network, then, is simply given by the eigenvector corresponding to the largest eigenvalue (also called the Perron eigenvalue) [39]. In brain network studies, the eigenvector centrality is a measure that approximates the centrality or the importance of a brain region to the corresponding functional network. Eigenvector centrality attributes a value to each voxel in the brain, such that a voxel receives a large value if it is strongly correlated with many other nodes that are themselves central within the network. A brain region has higher eigenvector centrality if its neighbors are also highly central. It has been demonstrated that eigenvector centrality is a computationally efficient tool for capturing intrinsic neural architecture on a voxel-wise level [40].

For a matrix $\mathbf{A} \in R^{N \times N}$, a number $\lambda$ is an eigenvalue if, for some vector $\vec{c} \neq 0$ [39],

$$
\vec{A} \vec{c}=\lambda \vec{c} .
$$

Here, the centrality vector $\vec{c}$ is the eigenvector of the adjacency matrix $\mathbf{A}$ associated with the eigenvalue $\lambda$. In general, eigenvectors give the direction of spread of data, while the eigenvalue is the intensity of spread in a particular direction or of that respective eigenvector. Given the weighted adjacency matrix $\mathbf{A}$ of network $G$, it is wise to choose the largest eigenvalue, $\lambda_{\max }$, in the absolute value of matrix. By virtue of the Perron-Frobenius theorem [39], this choice implies that if the graph is strongly connected, then the eigenvector solution $\vec{c}$ is both unique and positive.

\section{Simulation results}

\subsection{Synchronization measures vs. linear mixing}

Fig. 5 shows the mean synchronization (or coupling degree) obtained by various synchronization measures as a function of coupling strength $K$ and degree of overlap. Note that each mean value was calculated by averaging over all possible pairs of 64 modeled EEG time series in the Kuramoto model.

Theoretically, the global coupling exhibits a critical value $K_{c}$, for which the network exhibits a synchronization transition between an incoherent motion of the oscillators $\left(K<K_{c}\right)$ and partial synchrony $\left(K \geq K_{c}\right)$. In the case of an infinite number of oscillators, the network synchrony increases sharply from zero, below $K_{c}$, to a positive value. However, for finite-sized networks, a similar but smoother transition is observed in the behavior of the coupling. As mentioned earlier, in this work we set $\gamma=1$ which resulted in $K_{c}=2$. Hence, theoretically, we expected that the synchronization measures should start with zero when $K<2$, show a bifurcation or sharp peak at $K=2$, and then ascend continuously for $K>2$. As shown in Fig. 5, the ascending behavior of synchronization as a function of $K$ was observed for all measures. The non-zero values below $K_{c}$ merely reflect fluctuations in the simulation due to a finite number of oscillators. The relative increase in synchronization started at $K_{c}=2$ for the ImPC, PC, and PLI measures that were exactly the same as the theoretical value. For the rest of the measures, the sharp increase started at lower values than the analytically expected value.

In the case in which $i_{0}=0$, the linear CC measure (see Fig. 5(a)) started with a small value when $K=0$ and stayed at relatively low levels for $K<1$. The CC approached full synchronization around $K=5$. By switching on the linear mixing among the time series, the entire curve of the CC was shifted toward a higher level for all $K<5$. Also, increasing the level of overlap among the channels from $i_{0}=4$ to $i_{0}=8$ led to larger values for the synchronization only when $K<2$. The CC showed full synchronization for the overlapping cases when $K>2$. Therefore, we can conclude that the CC measure was sensitive to true changes in coupling strength and correctly predicted a small value for $K=0$. However, it did not conform well to theory, as it showed a peak in the synchronization around $K=1$ instead of the theoretically expected value $K_{c}=2$. Furthermore, the CC was quite sensitive to the VC effect that changed the predicted synchronization as a function of $K$.

The Coh measure overestimated the synchronization for cases with low coupling strength (i.e. $K<1$, see Fig. 5(b)). The overlap between the channels showed an upward displacement of the Coh curve, especially for $K>2$. Similar to the CC, we conclude that the Coh measure was sensitive to the spurious influence of common sources. Interpreting the results of the SL measure (see Fig. 5(c)) is difficult due to the fluctuating behavior of the curve. However, the large fluctuations reflect the fact that the measure cannot be sufficiently adopted to the broad frequency spectrum of the Kuramoto model. It can be seen that with high overlap of the EEG signals (i.e., $i_{0}=8$ ), an upward shift in the coupling degree was observed for $K<1.5$.

The results for ImPC (see Fig. 5(d)) showed that in the absence of VC, ImPC started at a very small value for $K=0$ and almost remained at this low level for $K<2$. By increasing the coupling strength to values higher than 2 , synchronization increased but never reached values higher than 0.22 , even for a very high coupling strength $K$. Therefore, we can say that the ImPC systematically underestimated the true coupling strength in the model. By switching on the linear superposition, ImPC increased for $K \leq 2.5$ and decreased for $K>2.5$. Hence, the effects of linear mixing further decreased the modest sensitivity of ImPC to the increase in coupling strength.

In the absence of overlap, the PC (see Fig. 5(e)) can capture the true amount of correlation, since it is directly linked to the formulation of the Kuramoto model. Hence, this method can be chosen as a standard measure to validate other methods only when $i_{0}=0$. It can be seen that the PC stayed at relatively small values for $K<2$ (see Fig. 5(d)), then showed a bifurcation at the critical level of $K=K_{c}=2$ and furthermore increased continuously for $K>2$. The PC averaging at $\sim 0.85$ 


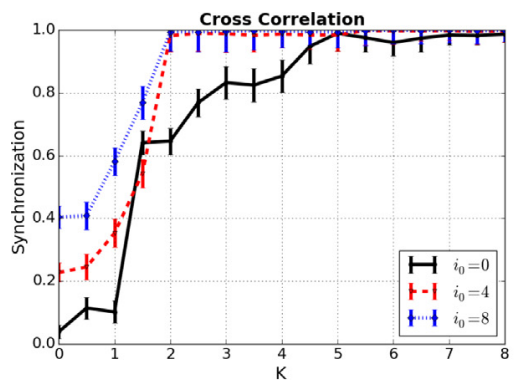

(a)

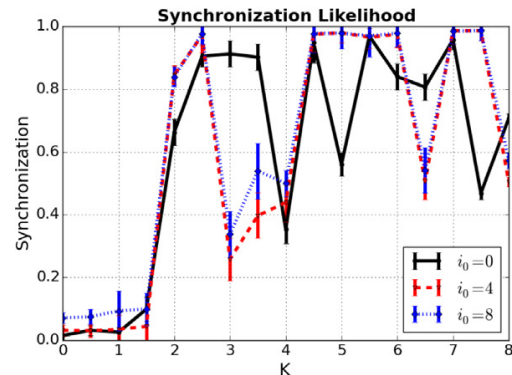

(c)

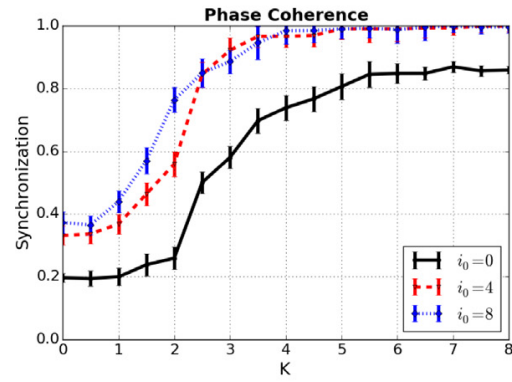

(e)

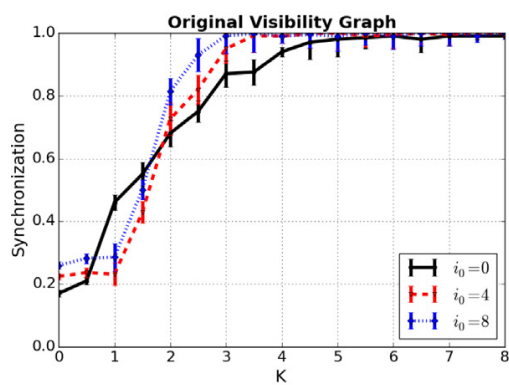

$(\mathrm{g})$

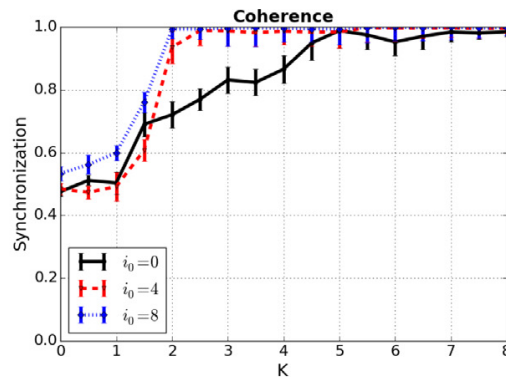

(b)

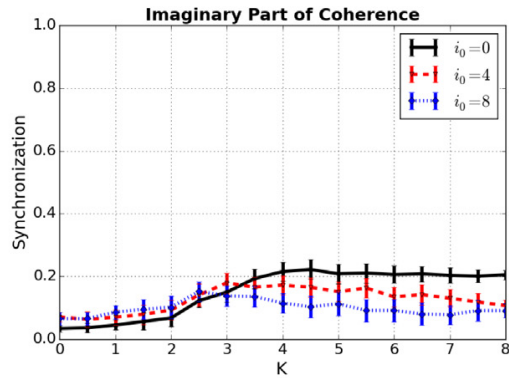

(d)

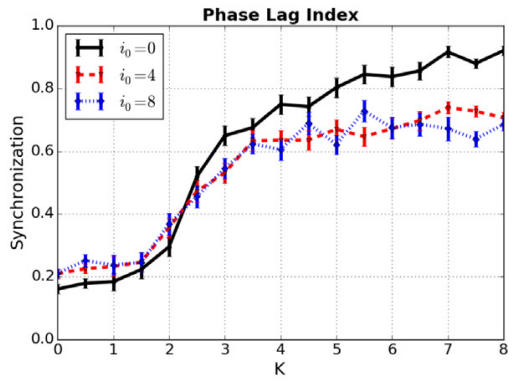

(f)

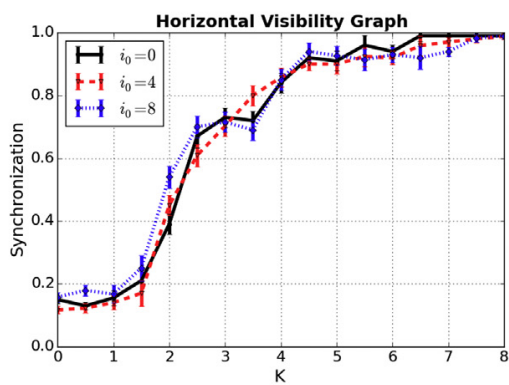

(h)

Fig. 5. Mean synchronization (or coupling degree) averaged over all possible pairs of 64 modeled EEG channels as a function of coupling strength $K$ in the Kuramoto model for different overlapping between subsequent EEG channels using (a) the CC, (b) the Coh, (c) the SL, (d) the ImPC, (e) the PC, (f) the PLI, (g) the OVG and (h) the HVG synchronization measures.

for $K>5$ meant that a considerable number of oscillators were not yet synchronized. If the coupling strength increased further $(K>8)$, clusters progressively merged to form bigger clusters, finally merging into a single cluster that resulted in full synchronization. By changing the overlap between time series from $i_{0}=0$ to $i_{0}=4$, the entire PC curve was shifted 


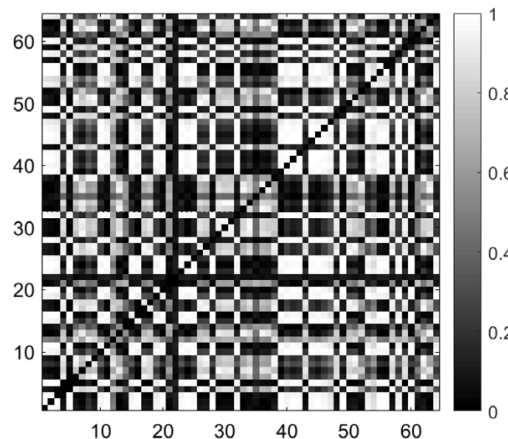

(a)

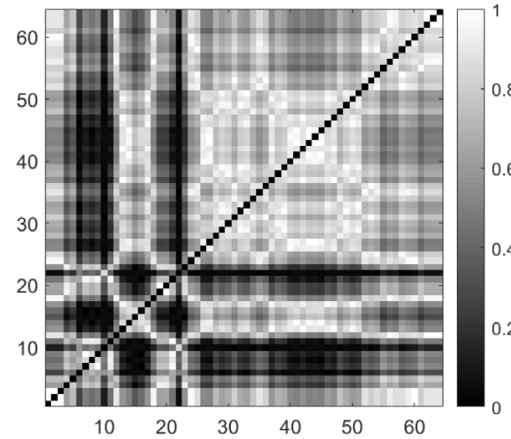

(b)

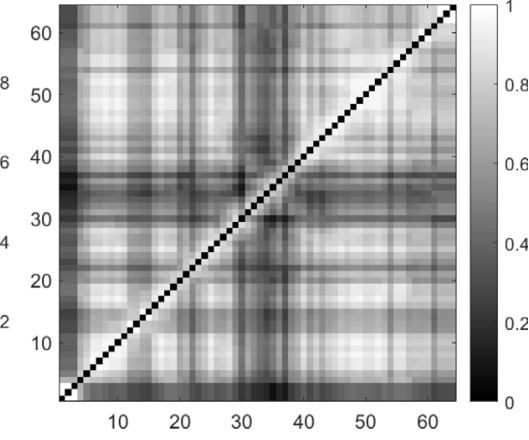

(c)

Fig. 6. Averaged correlation (or adjacency) matrix for (a) $i_{0}=0$, (b) $i_{0}=4$, and (c) $i_{0}=8$ when applying the HVG synchronization algorithm on the Kuramoto model with $K=2.5$.

towards a higher level for all values of coupling strength. Also, the relative increase in PC started at $K<2$. Increasing the level of overlap $i_{0}=4$ to $i_{0}=8$ showed an upward displacement of the curve, but only for values of $K<2.5$. For $i_{0}=8$, the relative increase in PC started at even lower values of $K$ compared to the case in which $i_{0}=4$. Therefore, it can be concluded that the PC was sensitive to true changes in coupling strength $K$ and was quite sensitive to spurious influences of linear mixing, which changed both the absolute values as well as the qualitative behavior of PC as a function of $K$.

The PLI (see Fig. 5(f)) showed low values for $K<2$ for $i_{0}=0$ and increasing values for higher $K$. This behavior is consistent with the theory. By adding the effect of VC, the PLI increased slightly for $K<2.5$ and decreased for $K>2.5$. It is clear that the PLI underestimated the true level of synchronization for high values of $K$ in cases with the effect of linear mixing. There was no clear difference between cases $i_{0}=4$ and $i_{0}=8$. Thus, PLI showed the expected increase as a function of $K$, but compared with PC or CC, it was less sensitive to the spurious influence of common sources.

In the case of no overlap, both the OVG and the HVG measures showed very good performance for $K<K_{c}$ (see Figs. $5(\mathrm{~g}$ ) and $5(\mathrm{~h})$ ). Both measures predicted small values until $K$ reached a critical value $1.5<K<2$, and then rapidly increased toward the asymptotic value of 1 . By comparing the OVG and HVG results with the PC curve at $i_{0}=0$, it is clear that the OVG and HVG measures predicted full synchronization for $K>5$ and $K>7$, respectively, which means overestimated correlations.

By adding the effect of linear mixing $\left(i_{0}=4\right)$, the OVG increased slightly for $K \leq 0.5$ and for $K \geq 1.5$ as well. A decrease in the level of synchronization was observed when $0.5<K<1$. Increasing the level of overlap $i_{0}=4$ to $i_{0}=8$ showed more displacement of the OVG curve. The HVG showed good performance against the spurious influence of common sources, as displacement in the HVG curve was very small compared to the other schemes. Therefore, we can conclude that both the OVG and the HVG synchronization measures had fairly good capability to predict the true synchronization between time series, for a wide range of coupling strengths, affected by the linear mixing. However, the HVG had a better capability, was superior to the other measures, and is very promising for estimating the synchronization among real EEG brain signals, especially when the coupling among brain regions is weak.

Fig. 6 shows the correlation matrix between 64 pairs of oscillators for $K=2.5$ and different overlapping parameters when the HVG synchronization measure was applied. The values of this matrix describe the amount of synchronization (or coupling degree) between oscillators. Each correlation matrix is equivalent to a weighted graph (or network) that is used for calculating network measures. It is worth mentioning that a weighted graph is visualized either as a correlation matrix or a node-link diagram. In Fig. 6, the weighted graph is visualized as a correlation matrix, as the weight of connections is mapped to the color of the corresponding cell. In Fig. 7, the node-like diagram of the weighted graph corresponding to Fig. 6(b) is shown. Again, the colors represent the weight of the connections.

\subsection{Effect of linear mixing on network measures}

The mean clustering coefficient of the network calculated by the selected synchronization measures is shown in Fig. 8(a) to $8(\mathrm{~h})$. Theoretically, for a weakly coupled network, the clustering coefficient is 0 or very close to 0 . The value of the clustering coefficient reaches 1 for a fully coupled network. Therefore, in our study, we expected to get a small value for the clustering coefficient when $K \rightarrow 0$ that was evident in the results calculated by all of the synchronization measures except the Coh. By increasing coupling strength (i.e., for $K>K_{c}$ ), the time series became more and more synchronized as a complete network is formed, in which each link had a weight equal to 1 .

The trend of the clustering measure in the presence of the overlapping effect depends on the selected synchronization measure. According to the results of Fig. 8, we can say that the clustering coefficient was not sensitive to the overlap for a wide range of coupling strengths if the PLI or HVG were applied as the synchronization measures. For weakly coupled networks 


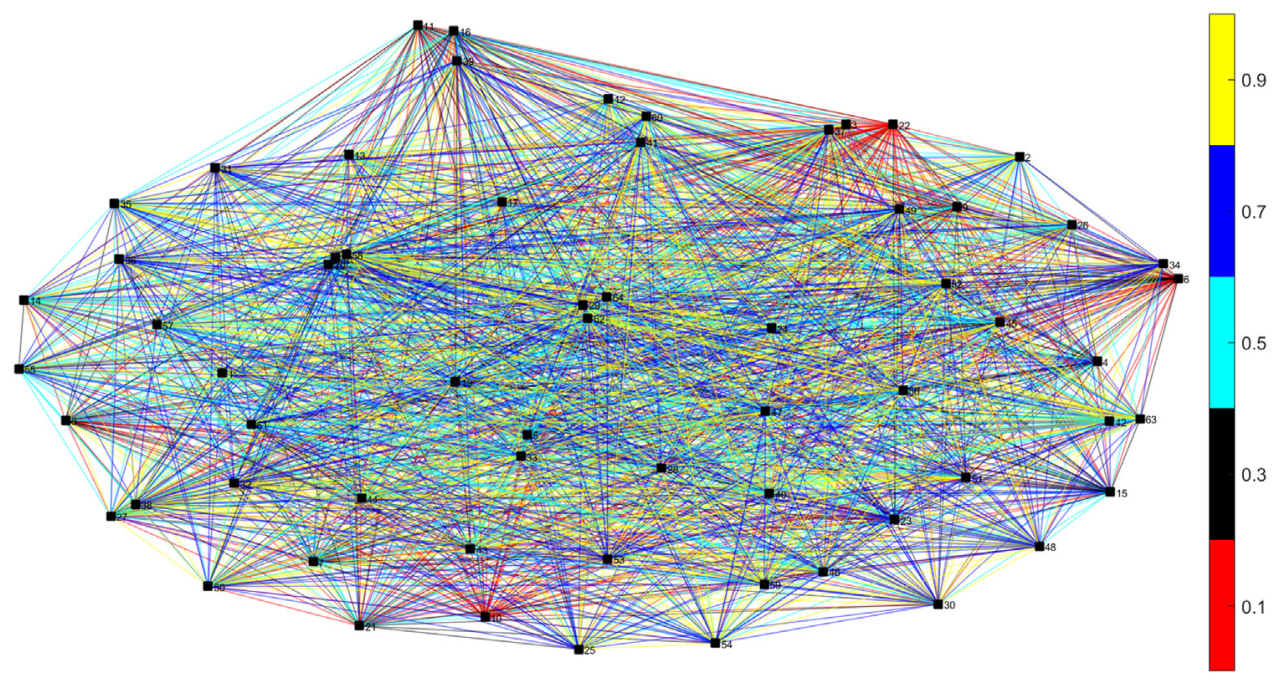

Fig. 7. The node-like diagram of the weighted graph corresponding to Fig. 6(b).

(i.e., for $K<K_{c}$ ), the clustering coefficients calculated by the SL, ImPC, and the OVG were not affected by the overlapping effect either. Therefore, the clustering coefficient can be selected as a measure to analyze a functional brain network affected by the VC if one of the above-mentioned synchronization measures are applied, depending on the coupling strength.

The behavior of the betweenness centrality calculated by various synchronization measures is shown in Fig. 9. As mentioned earlier, the betweenness centrality measures the extent to which a node lies on paths between other nodes. Theoretically, for a weakly coupled network (i.e., $K \rightarrow 0$ ) we should obtain a large value for the mean betweenness centrality. The reason is that, due to the weak coupling, there is no direct link between some nodes, and as a result, some other nodes may always lie on paths between many other pairs of vertices. Nodes with high betweenness usually have significant influence within a network by virtue of their control over information passing between others; their removal from the network will most likely disrupt communication between other vertices, because they lie on the largest number of paths taken by messages. By increasing the coupling strength to larger values (i.e., $K>K_{c}$ ), the averaged betweenness centrality approaches 0 , because the network becomes a complete network in which all pairs of vertices are connected to each other with a direct link or path. The betweenness values calculated by all of the selected synchronization measures, except the Coh, show similar behavior, i.e., starting from a large value when $K=0$ and approaching 0 by increasing the coupling strength.

Generally, by increasing the overlap, the betweenness centrality should decrease if the synchronization measure is sensitive to linear superposition. Our study showed that the betweenness centrality remained almost completely insensitive to the overlapping effect for all $K$ values if the SL was chosen as the synchronization measure. Furthermore, the ImPC is a good choice for calculating this network measure for low and moderate coupling strengths (i.e., $K \leq 2.5$ ). For fully coupled systems, the PLI method could be a suitable choice for calculating the betweenness centrality in the presence of the overlapping effect.

The largest eigenvalue and the eigenvector centrality of the network versus the coupling strength are shown in Figs. 10 and 11 , respectively. Generally, in the absence of the mixing effect, by increasing the coupling strength, the network becomes denser, and all nodes are connected to each other with strong weights. Therefore, the network approaches a 63-regular graph that results in the largest eigenvalue being equal to 63 for large enough coupling strengths. This behavior was observed in the results of Fig. 10 as the largest eigenvalues, calculated by all the synchronization measures, increased as the coupling strength increased, and finally approached 63 for high values of $K$. Again, the behavior of the largest eigenvalues was quite similar to the coupling degrees for each synchronization measure. These results show that the largest eigenvalues computed with the PLI and coupling degrees by the OVG and the HVG were not very sensitive to the effects of linear mixing. Furthermore, for systems with weak coupling (i.e., $K \leq K_{c}$ ), the largest eigenvalue calculated by the SL and the ImPC were insensitive to the overlapping effect. In other methods, when the overlapping effect increased, the largest eigenvalues increased as a result. As we pointed out earlier, the eigenvector centrality value of a $d$-regular graph is a constant vector. This is observed in Fig. 11, demonstrating that in the absence of linear mixing, the eigenvector centrality approached $\sim 0.125$ for all highly coupled cases. By switching on the overlapping effect, the values of the eigenvector centrality in weakly coupled cases grew and approached 0.125 . It can be seen that the eigenvector centrality calculated by the Coh method was constant for all values of $K$, whereas the eigenvector centrality calculated by all other methods started from a lower value and then rose to its final value by increasing the coupling strength.

As shown in Fig. 5(b), the Coh method overestimated the synchronization when $K \rightarrow 0$. Hence, its corresponding largest eigenvalue and the eigenvector centrality were overestimated as well. However, it can be seen that the eigenvector centrality 


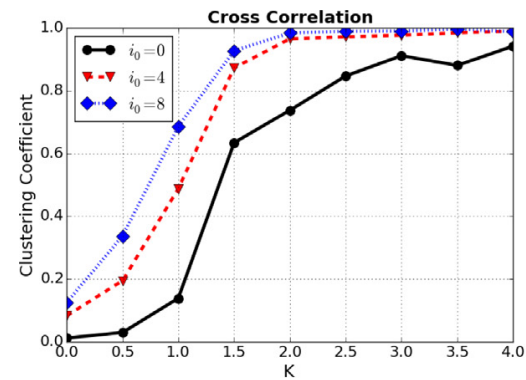

(a)

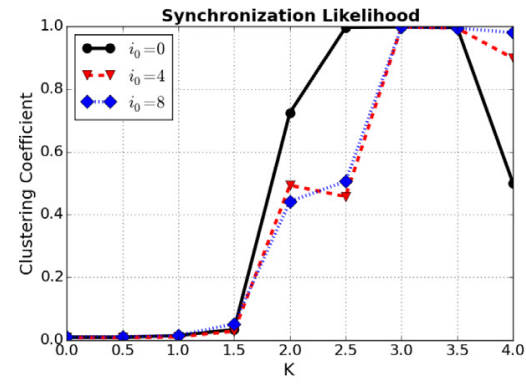

(c)

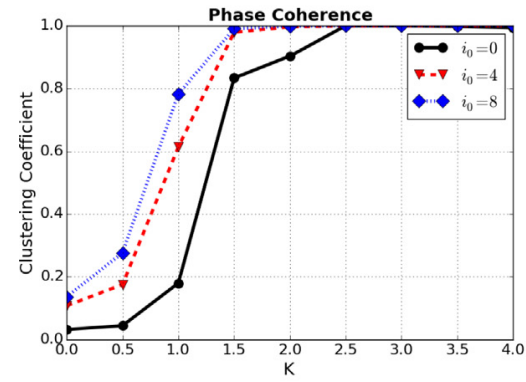

(e)

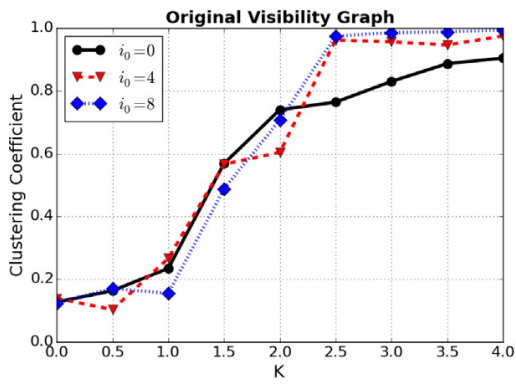

$(\mathrm{g})$

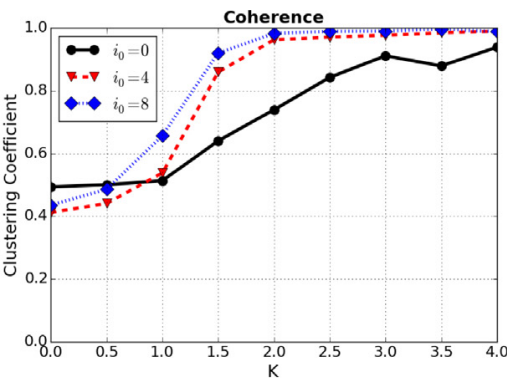

(b)

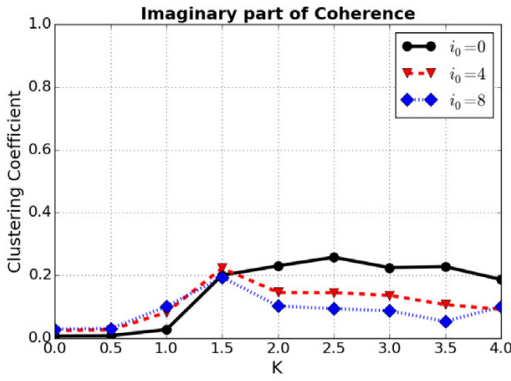

(d)

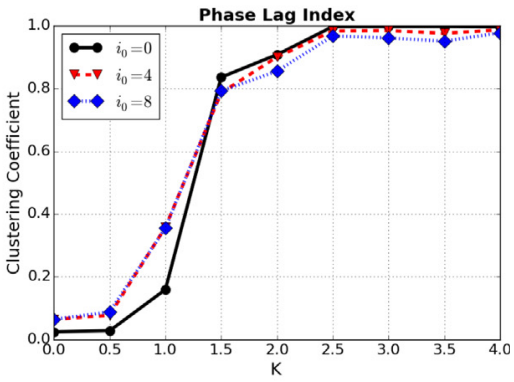

(f)

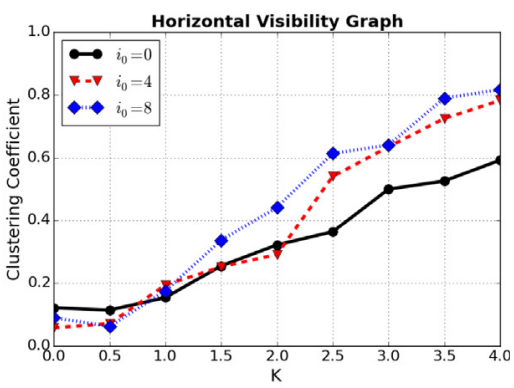

(h)

Fig. 8. The clustering coefficient value of network as a function of coupling strength $K$ in the Kuramoto model with 64 oscillators for different overlapping between subsequent EEG channels using (a) the CC, (b) the Coh, (c) the SL, (d) the ImPC, (e) the PC, (f) the PLI, (g) the OVG, and (h) the HVG measure.

calculated by the Coh was not sensitive to the effects of linear mixing. A similar behavior was also observed for this network measure if it was calculated by the OVG. Generally, the eigenvector centrality values calculated by all other synchronization measures were not sensitive to the overlap in time series if $K>K_{c}$. 


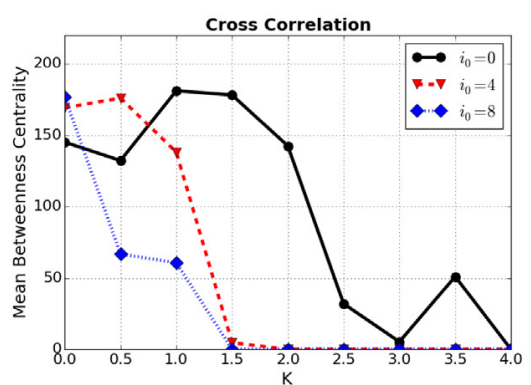

(a)

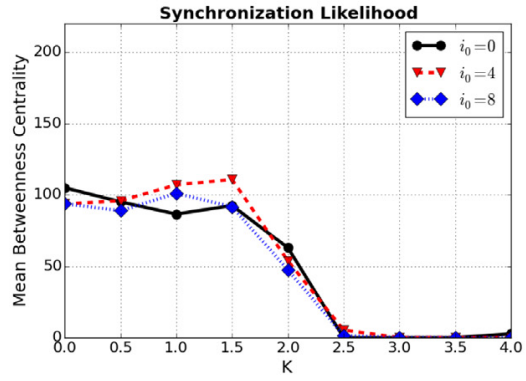

(c)

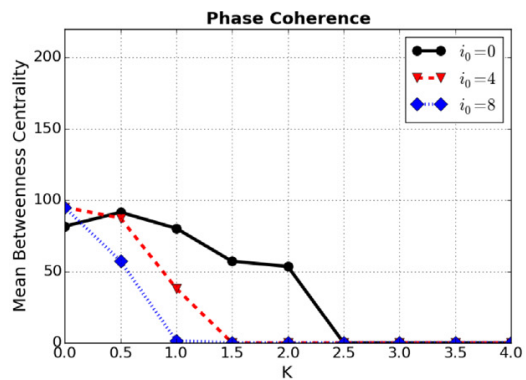

(e)

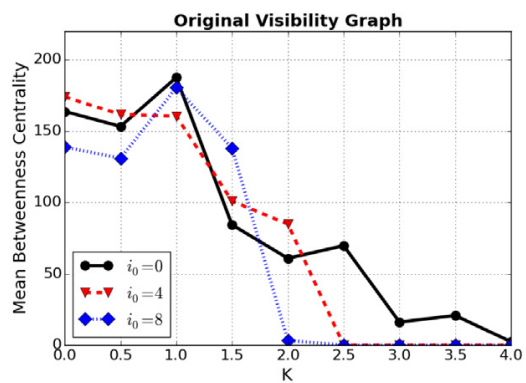

(g)

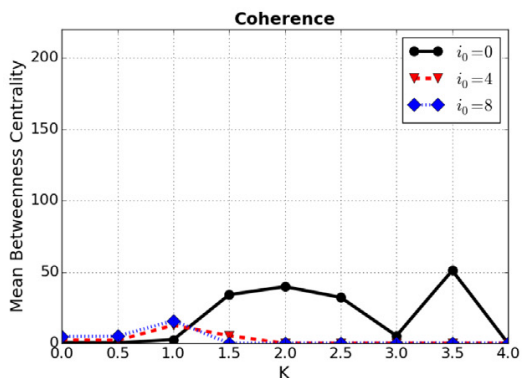

(b)

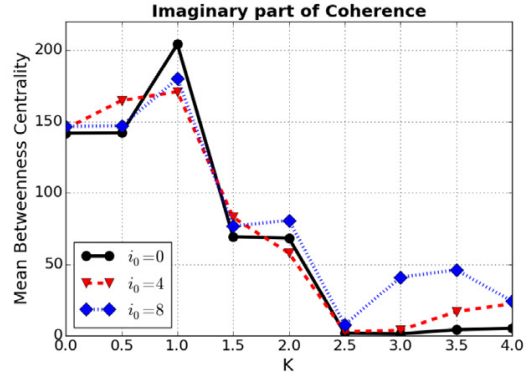

(d)

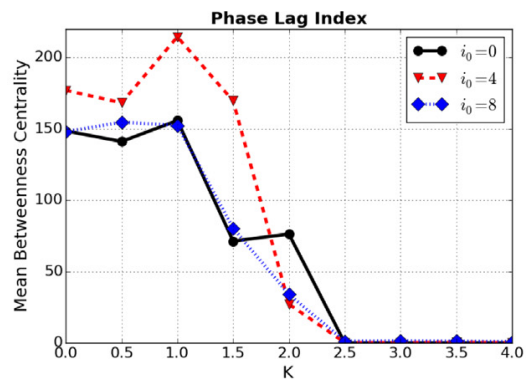

(f)

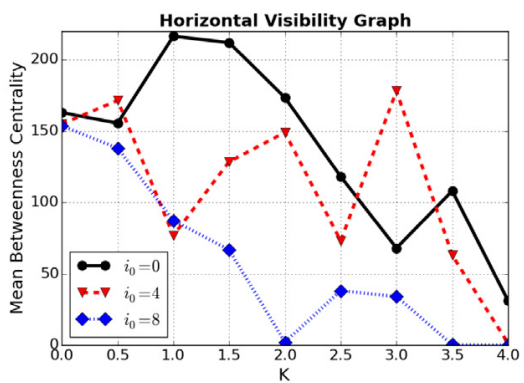

(h)

Fig. 9. The mean betweenness centrality of the network as a function of coupling strength $K$ in the Kuramoto model with 64 oscillators for difference overlapping between subsequent EEG channels using (a) the CC, (b) the Coh, (c) the SL, (d) the ImPC, (e) the PC, (f) the PLI, (g) the OVG, and (h) the HVG measure.

\section{Discussion}

We used the Kuramoto model of coupled phase oscillators to artificially generate EEG time series. Models of coupled oscillators have been widely used to understand the behavior of various systems in neurology as well as other disciplines. 


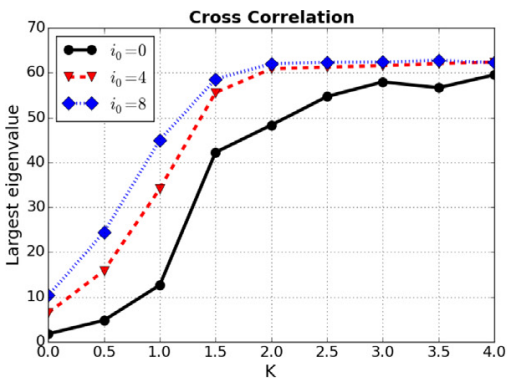

(a)

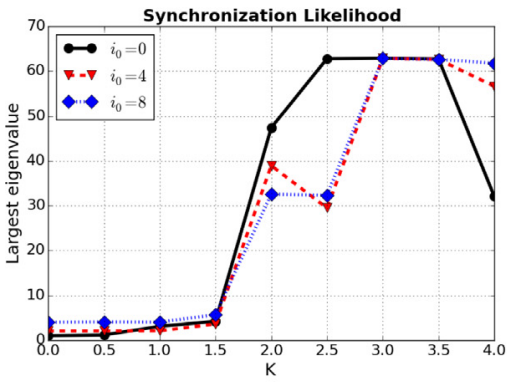

(c)

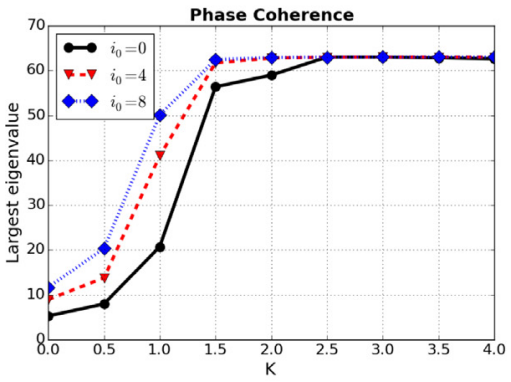

(e)

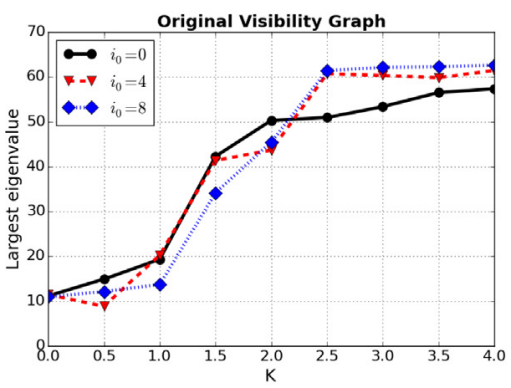

$(\mathrm{g})$

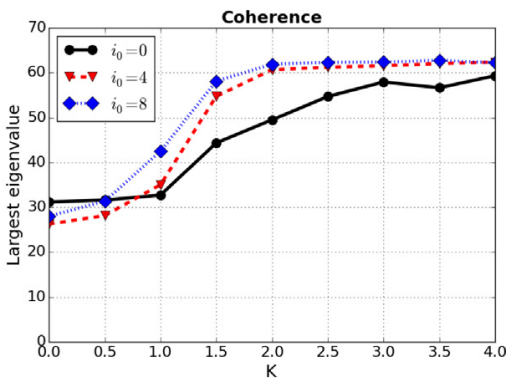

(b)

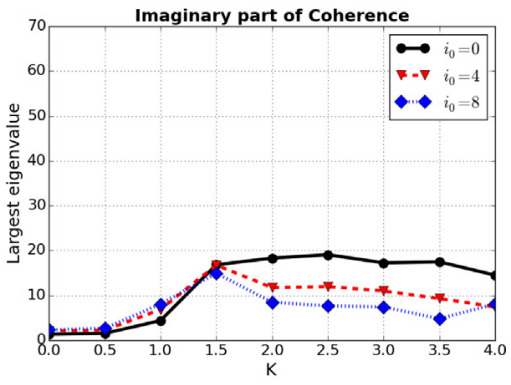

(d)

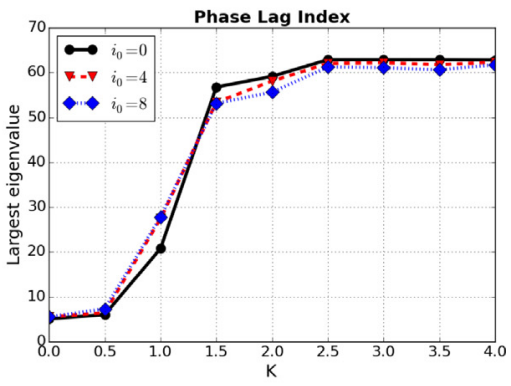

(f)

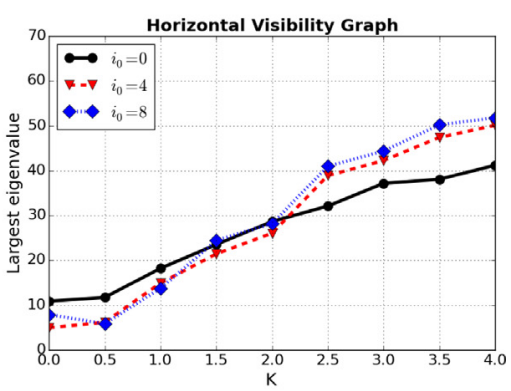

(h)

Fig. 10. Largest eigenvalue of each brain network as a function of coupling strength $K$ in the Kuramoto model with 64 oscillators as a function of overlap between subsequent EEG channels using (a) the CC, (b) the Coh, (c) the SL, (d) the ImPC, (e) the PC, (f) the PLI, (g) the OVG, and (h) the HVG measure.

These systems have been observed to synchronize themselves to a common frequency when the coupling strength between the oscillators is increased [16,41,42]. In order to figure out the collective phenomena when finite-range interactions are considered, it is of fundamental importance to study and understand the case of nearest neighbor interactions, which is the simplest form of local interactions. In this context, the original Kuramoto model with nearest neighbor coupling in a ring topology is a reasonable method to describe the behavior of coupled systems with local interactions. 


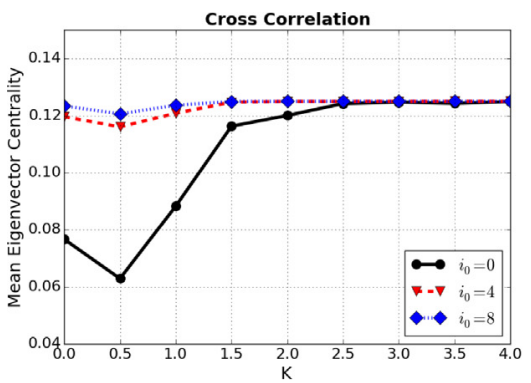

(a)

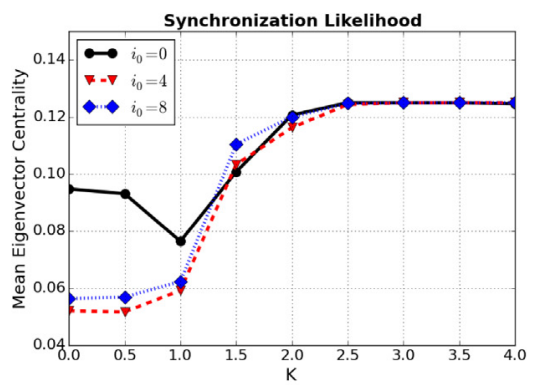

(c)

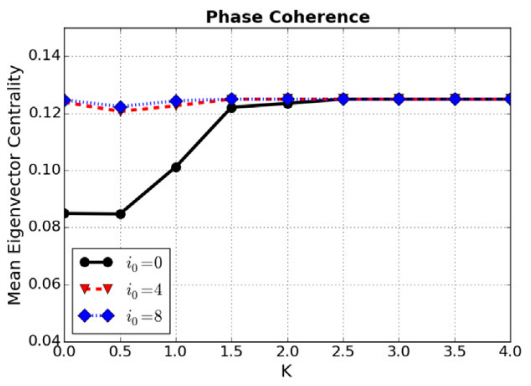

(e)

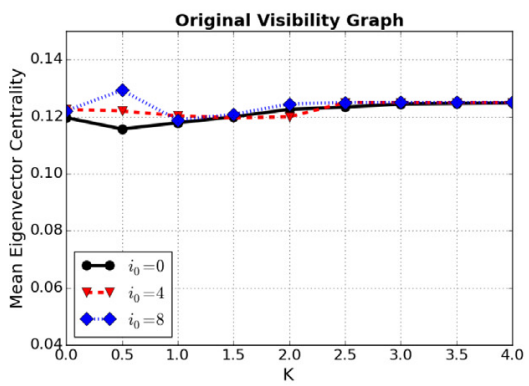

(g)

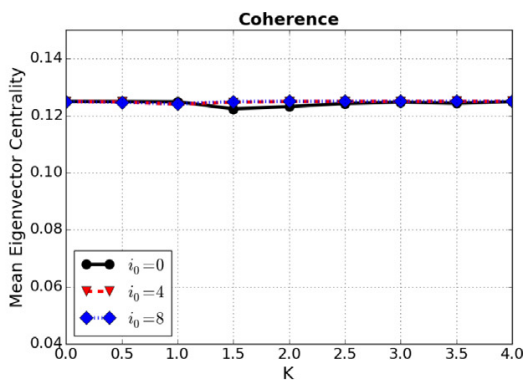

(b)

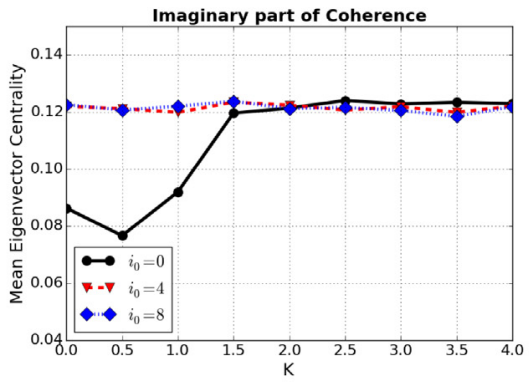

(d)

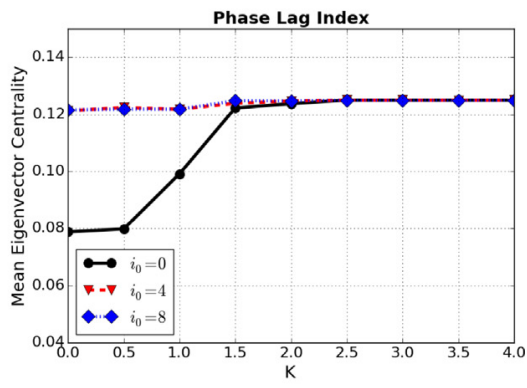

(f)

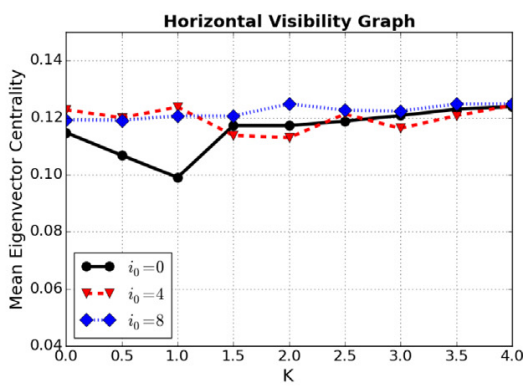

(h)

Fig. 11. The mean eigenvector centrality of each brain network as a function of coupling strength $K$ in the Kuramoto model with 64 oscillators as a function of overlap between subsequent EEG channels using (a) the CC, (b) the Coh, (c) the SL, (d) the ImPC, (e) the PC, (f) the PLI, (g) the OVG, and (h) the HVG measure.

When using the Kuramoto model, one assumes that the activity of a local system (e.g., a neuron or cortical area) can be sufficiently represented by its circular phase alone as long as the coupling is weak and the subsystems are nearly identical. Although dynamics restricted to a scalar phase measure for each subsystem may seem highly restrictive, Kuramoto [16] 
showed that interactions among these entities, which collectively constitute a dynamical structure at the next-to-coarsest spatial scale, are then introduced by a simple algebraic form that captures the essential characteristics of their exchanges, such as post-synaptic transmembrane perturbation. This phase reduction approach has now become a standard technique in computational neuroscience [43-47], which has offered a direct link between computational models of neurons and models of weakly coupled phase oscillators, permitting a variety of insights into the relationship between the phase response curve and the nature of synchronous activity at the neuronal level.

In the brain, the axons, or outputs, of the neurons connect via synapses to the dendrites, or inputs, of other neurons. The synapses secrete electrochemical neurotransmitters to the dendrites; these neurotransmitters can have an excitatory or an inhibitory influence on the firing of the neurons they connect to [48]. From this simple representation, we can see that the Kuramoto model has some similarities and some differences to the observed behavior in the brain. The model describes each oscillator as having a natural frequency, which could be seen as corresponding to the natural firing rate of a neuron [49-51]. Therefore, the Kuramoto model could be a fair approximation of a small network of densely connected neurons as a purely phenomenological model, which allows analytical studies of synchronization phenomena. However, it is certainly not true for large populations of neurons distributed across the cortical sheet. In order to make the system more neurobiologically reasonable, some less restrictive assumptions should be applied with regards to connection topology and interaction functions. Breakspear et al. [47] reviewed this simplified model of coupled phase oscillators in the context of models of complex neurobiological systems and found that it captured the core mechanisms of neuronal synchronization and a broad repertoire of rich, nontrivial cortical dynamics.

By using the Kuramoto model, we investigated the sensitivity of various selected synchronization measures to the linear mixing effect. The CC measure is the oldest and most commonly used measure of synchronization of two time series. The value of the time lag is taken as an estimation of the delay between the time series, under the implicit assumption that they are linearly related. However, this delay cannot be directly regarded as a measure of the propagation time of the electrical signal in the brain [52]. The CC has been effective to study neuronal systems containing dominant unidirectional interactions, as the time-lagged maximal correlation and the magnitude of correlation can be informative to information flow between brain areas [53]. Our results showed that the CC measure was sensitive to the linear mixing effect, which is in line with findings that the presence of several kinds of artifacts might cause a loss of information and alteration in the results of CC analysis [54]. However, the CC remains one of the most widely used measures to reveal the correlation among EEG time series in brain studies.

The Coh quantifies linear correlations between two time series in the frequency domain. The Coh is sensitive to both phase and amplitude relationships between the time series. Therefore, the relative importance of amplitude and phase covariance in this index is not altogether clear [55,56]. It should be mentioned that by using the Coh measure alone, we could not determine the directionality of the network interaction. Also, the exact amplitudes of the network interaction are not the same as region-to-region coherence amplitudes. The Coh provides a global estimate of all important regions of network activity regardless of source amplitudes. Thus, this measure is an appropriate choice for estimating correlation among long time series that persist for extended durations [57].

The main weakness of the Coh measure is that it is strongly affected by VC. The ImPC is aimed at eliminating all sources of extraneous coherence that are a consequence of instantaneous activity [26]. Hence, the ImPC captures true source interactions at a given time lag. The ImPC has gained traction during the past years in EEG connectivity studies [58]. An important property of the ImPC is that its non-vanishing finite value cannot be caused by a linear mixing of uncorrelated sources and thus reflects true interactions of the sources underlying the two time series [19].

However, in some situations, the functional connectivity estimate based on ImPC becomes negligible, even in the presence of a significant true interaction, e.g., the phase difference between two time series is near 0 or $\pi$ [19]. To overcome this issue, the PLI was introduced by Stam et al. [19]. The PLI is an alternative measure of phase synchronization reflecting the strength of the coupling by looking for consistent, non-zero phase lag between two time series. The PLI measures phase synchronization based on the asymmetry of the distribution of instantaneous phase differences between two time series, which is determined using the analytical signal based on the Hilbert transform. True interaction between two neural sources results in a coherent phase relationship between their corresponding time series at a value different from 0 and $\pi$. The idea behind the PLI is to discard phase differences that center around $0 \mathrm{mod} \pi$, which can be done by defining an asymmetry index for the distribution of phase differences when the distribution is centered around a phase difference of 0 [59]. Our simulations showed that the PLI was able to detect synchronization in the Kuramoto model with moderate $K$. With very high values of coupling strength, the mean phase difference between all the oscillators vanishes, which might explain why the PLI did not reach a value of one. Although the PLI was not insensitive to linear mixing, these effects were clearly smaller than other measures. This readily suggests that the PLI could be a more reliable measure of true synchronization compared to other measures such as CC, Coh, or ImPC. However, it is worth mentioning that the sensitivity of the PLI to noise is hindered by the discontinuity in the measure, as small perturbations may turn phase lags into leads and vice versa. In other words, in case of noisy time series, where phase difference values close to zero may change from leads to lags due to the presence of noise, the PLI is biased and loses some ability to detect changes in phase synchronization, especially in cases of weak coupling [60]. This problem can be solved by using the weighted formulation of PLI, in which not only the phase but also the amplitude of the imaginary part of the cross spectrum is taken into account [60].

If one is only interested in the relationship between the phases without any influence of the amplitudes, then other methods such as PC should be applied. It is well known that the instantaneous phases of two coupled time series may 
be strongly synchronized even if their amplitudes remain independent, a state referred to as phase coherence [61]. The PC between two time series appears when time series are coupled in a broad range of structures. Hence, time frequency analysis of phase synchronization is popular in research on brain network identification. The PC is better used for short duration events where phase is used to determine how much the two recording sites are interacting within a very narrow time window (milliseconds). However, our study showed that the PC was strongly affected by the overlapping effect, although it still increased with increases in coupling strength. Hence, absolute values of PC cannot be interpreted in the context of unknown effects of VC, while changes in PC between experimental conditions could still reflect changes in coupling [19].

The SL measure is a statistical likelihood with a logical decision-making process that determines whether the patterns are similar or not compared with a threshold, disregarding how much similarity there is in the patterns. However, the similarity is not considered in the decision-making process, which may affect the reliability of the method. It has been shown that the SL is robust to change in the sampling frequency of the data and tracks recurrence of complex patterns with broad frequency content [29]. This measure can be computed in a time-dependent way, making it suitable for the analysis of non-stationary data.

The synchronization measures based on the visibility graph were used as a new way to detect synchronization between time series. By carrying out the VG algorithm, the data are mapped from the time domain to the graph domain, which allows for the dynamics of the time series to be studied via the topology of the graph; subsequently, the structure of the time series can be analyzed through the set of tools developed in the graph theory. Therefore, the information on time series is obtained just by analyzing the characteristics of the corresponding visibility graph. This mapping gains some practical interest as it opens the possibility of analyzing a time series from an alternative angle. In particular, it has been shown that both the structure of complex, irregular time series and the nontrivial ingredients of its underlying dynamics are inherited in the topology of the visibility graphs, and therefore simple topological properties of the graphs can be used as time series features for description and automatic classification purposes [62]. Our results showed that the HVG and OVG algorithms had a fairly good ability to predict true synchronization between the time series affected by linear mixing. It is worth mentioning that the presented VG-based algorithms are computationally efficient to transform small-scale time series to visibility graphs, but it may take too much time to deal with very large time series. To transform a time series of size $N$, it is necessary to check all the $N(N-1) / 2$ pairs of time series nodes to determine whether each pair of nodes can see each other based on the defined geometrical criteria. Therefore, the total time complexity of the VG algorithm is $O\left(N^{2}\right)$. In other words, the algorithm takes $O\left(N^{2}\right)$ time to execute to detect synchronization between two time series for the time duration $N$.

\section{Conclusion}

Volume conduction is an important complication that may lead to spurious synchronization among correlated signals when attempting to find synchronization among EEG signals. In this paper, we examined the performance of various synchronization measures against linear mixing as a way to model the VC effect. The EEG signals were generated based on the Kuramoto model. Our study showed that the PLI, OVG, and HVG synchronization measures had good performance in detecting the onset of synchronization. These measures, not being very sensitive to the VC effect for a wide range of coupling strengths, can be used reliably for finding synchronization among real EEG signals for functional brain network studies.

\section{References}

[1] E. Bullmore, O. Sporns, Complex brain networks: graph theoretical analysis of structural and functional systems, Nat. Rev. Neurosci. 10 (3) (2009) 186-198.

[2] E. Bullmore, O. Sporns, The economy of brain network organization, Nat. Rev. Neurosci. 13 (5) (2012) 336-349.

[3] D. Ariely, G.S. Berns, Neuromarketing: the hope and hype of neuroimaging in business, Nat. Rev. Neurosci. 11 (4) (2010) $284-292$.

[4] Y. He, A. Evans, Graph theoretical modeling of brain connectivity, Curr. Opin. Neurol. 23 (4) (2010) 341-350.

[5] M. Rubinov, O. Sporns, Complex network measures of brain connectivity: uses and interpretations, Neuroimage 52 (3) (2010) 1059-1069.

[6] E.T. Bullmore, D.S. Bassett, Brain graphs: graphical models of the human brain connectome, Annu. Rev. Clin. Psychol. 7 (2011) $113-140$.

[7] T. König, L. Prichep, T. Dierks, D. Hubl, L. Wahlund, E. John, V. Jelic, Decreased eeg synchronization in alzheimer's disease and mild cognitive impairment, Neurobiol. Aging 26 (2) (2005) 165-171.

[8] P. Nunez, R. Silberstein, P. Cadusch, R. Wijesinghe, A. Westdorp, R. Srinivasan, A theoretical and experimental study of high resolution eeg based on surface laplacians and cortical imaging, Electroencephalogr. Clin. Neurophysiol. 90 (1) (1994) 40-57.

[9] B. He, L. Yang, C. Wilke, H. Yuan, Electrophysiological imaging of brain activity and connectivity challenges and opportunities, IEEE Trans. Biomed. Eng. 58 (7) (2011) 1918-1931.

[10] R. Srinivasan, P.L. Nunez, D.M. Tucker, R.B. Silberstein, P.J. Cadusch, Spatial sampling and filtering of EEG with spline laplacians to estimate cortical potentials, Brain Topogr. 8 (4) (1996) 355-366.

[11] S. Makeig, A.J. Bell, T.P. Jung, T.J. Sejnowski, Independent component analysis of electroencephalographic data, in: Advances in neural information processing systems, 1996, pp. 145-151.

[12] P.L. Nunez, R. Srinivasan, A.F. Westdorp, R.S. Wijesinghe, D.M. Tucker, R.B. Silberstein, P.J. Cadusch, Eeg coherency: i: statistics, reference electrode, volume conduction, laplacians, cortical imaging, and interpretation at multiple scales, Electroencephalogr. Clin. Neurophysiol. 103 (5)(1997) 499-515.

[13] J.-M. Schoffelen, J. Gross, Source connectivity analysis with MEG and EEG, Hum. Brain Mapp. 30 (6) (2009) 1857-1865.

[14] R. Srinivasan, P.L. Nunez, R.B. Silberstein, Spatial filtering and neocortical dynamics: estimates of eeg coherence, IEEE Trans. Biomed. Eng. 45 (7) (1998) 814-826.

[15] P.L. Nunez, R. Srinivasan, Electric Fields of the Brain: the Neurophysics of EEG, Oxford University Press, USA, 2006.

[16] Y. Kuramoto, Chemical Oscillations, Waves, and Turbulence, vol. 19, Springer Science \& Business Media, 2012.

[17] I.Z. Kiss, Y. Zhai, J.L. Hudson, Emerging coherence in a population of chemical oscillators, Science 296 (5573) (2002) 1676-1678. 
[18] L.R. Peraza, A.U. Asghar, G. Green, D.M. Halliday, Volume conduction effects in brain network inference from electroencephalographic recordings using phase lag index, J. Neurosci. Methods 207 (2) (2012) 189-199.

[19] C.J. Stam, G. Nolte, A. Daffertshofer, Phase lag index: assessment of functional connectivity from multi channel eeg and meg with diminished bias from common sources, Hum. Brain Mapp. 28 (11)(2007) 1178-1193.

[20] M. Lobier, F. Siebenhühner, S. Palva, J.M. Palva, Phase transfer entropy: a novel phase-based measure for directed connectivity in networks coupled by oscillatory interactions, Neuroimage 85 (2014) 853-872.

[21] R. Oostenveld, P. Fries, E. Maris, J.-M. Schoffelen, Fieldtrip: open source software for advanced analysis of meg, eeg, and invasive electrophysiological data, Comput. Intell. Neurosci. 2011 (2011) 1.

[22] M. Yu, A. Hillebrand, A.A. Gouw, C.J. Stam, Horizontal visibility graph transfer entropy (hvg-te): a novel metric to characterize directed connectivity in large-scale brain networks, NeuroImage (2017).

[23] N. Ahmadi, E. Carrette, A.P. Aldenkamp, M. Pechenizkiy, Finding predictive EEG complexity features for classification of epileptic and psychogenic nonepileptic seizures using imperialist competitive algorithm, in: 2018 IEEE 31st International Symposium on Computer-Based Medical Systems (CBMS), IEEE, 2018, pp. 164-169.

[24] G.E. Box, G.M. Jenkins, G.C. Reinsel, G.M. Ljung, Time Series Analysis: Forecasting and Control, John Wiley \& Sons, 2015.

[25] D. Gabor, Theory of communication. part 1: the analysis of information, J. Inst. Electr. Eng. 93 (26) (1946) 429-441.

[26] G. Nolte, O. Bai, L. Wheaton, Z. Mari, S. Vorbach, M. Hallett, Identifying true brain interaction from eeg data using the imaginary part of coherency, Clin. Neurophysiol. 115 (10) (2004) 2292-2307.

[27] F. Mormann, K. Lehnertz, P. David, C.E. Elger, Mean phase coherence as a measure for phase synchronization and its application to the eeg of epilepsy patients, Physica D 144 (3-4) (2000) 358-369.

[28] C. Stam, B. Van Dijk, Synchronization likelihood: an unbiased measure of generalized synchronization in multivariate data sets, Physica D 163 (3) (2002) 236-251.

[29] T. Montez, K. Linkenkaer-Hansen, B. Van Dijk, C. Stam, Synchronization likelihood with explicit time-frequency priors, Neuroimage 33 (4) (2006) $1117-1125$

[30] R.F. Betzel, M.A. Erickson, M. Abell, B.F. O’Donnell, W.P. Hetrick, O. Sporns, Synchronization dynamics and evidence for a repertoire of network states in resting eeg, Front. Comput. Neurosci. 6 (2012) 74.

[31] L. Lacasa, R. Toral, Description of stochastic and chaotic series using visibility graphs, Phys. Rev. E 82 (3) (2010) 036120.

[32] B. Luque, L. Lacasa, F. Ballesteros, J. Luque, Horizontal visibility graphs: exact results for random time series, Phys. Rev. E 80 (4) (2009) 046103.

[33] A.S. Campanharo, M.I. Sirer, R.D. Malmgren, F.M. Ramos, L.A.N. Amaral, Duality between time series and networks, PLoS One 6 (8) (2011) e23378,

[34] M. Ahmadlou, H. Adeli, Visibility graph similarity: a new measure of generalized synchronization in coupled dynamic systems, Physica D 241 (4) (2012) 326-332.

[35] N. Ahmadi, M. Pechenizkiy, Application of horizontal visibility graph as a robust measure of neurophysiological signals synchrony, in: Computer-Based Medical Systems (CBMS), 2016 IEEE 29th International Symposium on, IEEE, 2016, pp. 273-278.

[36] I. Antoniou, E. Tsompa, Statistical analysis of weighted networks, Discrete Dyn. Nat. Soc. 2008 (2008).

[37] L.d.F. Costa, F.A. Rodrigues, G. Travieso, P.R. Villas Boas, Characterization of complex networks: a survey of measurements, Adv. Phys. 56 (1) (2007) $167-242$.

[38] U. Brandes, C. Pich, Centrality estimation in large networks, Int. J. Bifurcation Chaos 17 (07) (2007) 2303-2318.

[39] P. Van Mieghem, Graph Spectra for Complex Networks, Cambridge University Press, 2010.

[40] G. Lohmann, D.S. Margulies, A. Horstmann, B. Pleger, J. Lepsien, D. Goldhahn, H. Schloegl, M. Stumvoll, A. Villringer, R. Turner, Eigenvector centrality mapping for analyzing connectivity patterns in fmri data of the human brain, PLoS One 5 (4) (2010) e10232.

[41] S.C. Manrubia, A.S. Mikhailov, et al., Emergence of Dynamical Order: Synchronization Phenomena in Complex Systems, World Scientific, 2004.

[42] H. Haken, Brain dynamics(synchronisation and activity patterns in pulse-coupled neural nets with delays and noise), in: Springer Series in Synergetics, Springer, 2002.

[43] G. Ermentrout, N. Kopell, Parabolic bursting in an excitable system coupled with a slow oscillation, SIAM J. Appl. Math. 46 (2) (1986) $233-253$.

[44] G. Ermentrout, N. Kopell, Oscillator death in systems of coupled neural oscillators, SIAM J. Appl. Math. 50 (1)(1990) $125-146$.

[45] J. Guckenheimer, P. Holmes, Nonlinear Oscillations, Dynamical Systems, and Bifurcations of Vector Fields, vol. 42, Springer Science \& Business Media, 2013.

[46] E. Brown, J. Moehlis, P. Holmes, On the phase reduction and response dynamics of neural oscillator populations, Neural Comput. 16 (4) (2004)673-715.

[47] M. Breakspear, S. Heitmann, A. Daffertshofer, Generative models of cortical oscillations: neurobiological implications of the kuramoto model, Front. Hum. Neurosci. 4 (2010) 190

[48] E.R. Kandel, J.H. Schwartz, T.M. Jessell, S.A. Siegelbaum, A.J. Hudspeth, et al., Principles of Neural Science, vol. 4, McGraw-hill, New York, 2000.

[49] R.R. Llinás, The intrinsic electrophysiological properties of mammalian neurons: insights into central nervous system function, Science 242 (4886) (1988) 1654-1664.

[50] B. Hutcheon, Y. Yarom, Resonance, oscillation and the intrinsic frequency preferences of neurons, Trends Neurosci. 23 (5) (2000) $216-222$.

[51] J. Gómez-Gardeñes, G. Zamora-López, Y. Moreno, A. Arenas, From modular to centralized organization of synchronization in functional areas of the cat cerebral cortex, PloS One 5 (8) (2010) e12313.

[52] E. Pereda, R.Q. Quiroga, J. Bhattacharya, Nonlinear multivariate analysis of neurophysiological signals, Prog. Neurobiol. 77 (1) (2005) 1-37.

[53] J.-M. Alonso, W.M. Usrey, R.C. Reid, Precisely correlated firing in cells of the lateral geniculate nucleus, Nature 383 (6603) (1996) 815.

[54] D. Simpson, A. Infantosi, D.B. Rosas, Estimation and significance testing of cross-correlation between cerebral blood flow velocity and background electro-encephalograph activity in signals with missing samples, Med. Biol. Eng. Comput. 39 (4) (2001) 428-433.

[55] J.P. Lachaux, E. Rodriguez, J. Martinerie, F.J. Varela, Measuring phase synchrony in brain signals, Hum. brain Mapp. 8 (4) (1999) 194-208.

[56] F. Varela, J.P. Lachaux, E. Rodriguez, J. Martinerie, The brainweb: phase synchronization and large-scale integration, Nat. Rev. Neurosci. 2 (4) (2001) 229.

[57] S.M. Bowyer, Coherence a measure of the brain networks: past and present, Neuropsych. Electrophys. 2 (1) (2016) 1

[58] L.G. Domínguez, J. Stieben, J.L.P. Velázquez, S. Shanker, The imaginary part of coherency in autism: differences in cortical functional connectivity in preschool children, PLoS One 8 (10) (2013) e75941.

[59] G. González, M. Van der Molen, G. Žarić, M. Bonte, J. Tijms, L. Blomert, C. Stam, M. Van der Molen, Graph analysis of eeg resting state functional networks in dyslexic readers, Clin. Neurophysiol. 127 (9) (2016) 3165-3175.

[60] M. Vinck, R. Oostenveld, M. Van Wingerden, F. Battaglia, C.M. Pennartz, An improved index of phase-synchronization for electrophysiological data in the presence of volume-conduction, noise and sample-size bias, Neuroimage 55 (4) (2011) 1548-1565.

[61] A. Pikovsky, M. Rosenblum, J. Kurths, J. Kurths, Synchronization: A Universal Concept in Nonlinear Sciences, vol. 12, Cambridge university press, 2003.

[62] L. Lacasa, B. Luque, F. Ballesteros, J. Luque, J.C. Nuno, From time series to complex networks: the visibility graph, Proc. Natl. Acad. Sci. 105 (13) (2008) 4972-4975. 